\title{
Robust Solution of Monotone Stochastic Linear Complementarity Problems ${ }^{1}$
}

\author{
Xiaojun Chen Chao Zhang \\ Department of Mathematical System Science, Faculty of Science and Technology, \\ Hirosaki University, Hirosaki 036-8561, Japan \\ Masao Fukushima \\ Department of Applied Mathematics and Physics, Graduate School of Informatics, \\ Kyoto University, Kyoto 606-8501, Japan
}

19 July 2005, Revised 26 March 2006

\begin{abstract}
We consider the stochastic linear complementarity problem (SLCP) involving a random matrix whose expectation matrix is positive semi-definite. We show that the expected residual minimization (ERM) formulation of this problem has a nonempty and bounded solution set if the expected value (EV) formulation, which reduces to the LCP with the positive semi-definite expectation matrix, has a nonempty and bounded solution set. We give a new error bound for the monotone LCP and use it to show that solutions of the ERM formulation are robust in the sense that they may have a minimum sensitivity with respect to random parameter variations in SLCP. Numerical examples including a stochastic traffic equilibrium problem are given to illustrate the characteristics of the solutions.
\end{abstract}

Key words. Stochastic linear complementarity problem; NCP function; expected residual minimization

\section{Introduction}

The linear complementarity problem (LCP) is to find a vector $x \in R^{n}$ such that

$$
A x+p \geq 0, x \geq 0, x^{T}(A x+p)=0,
$$

where $A \in R^{n \times n}$ and $p \in R^{n}$. This problem is generally denoted as $\operatorname{LCP}(A, p)$. The LCP has a significant number of applications in engineering and economics $[4,6,9]$. In practice, due to several types of uncertainties such as weather, material, trade, loads, supply, demand, cost, etc., the data in the LCP can only be estimated based on limited information. Suppose that $M(\omega) \in R^{n \times n}, q(\omega) \in R^{n}$, for $\omega \in \Omega \subseteq R^{m}$, are random quantities on a probability space $(\Omega, \mathcal{F}, \mathcal{P})$, where the probability distribution $\mathcal{P}$ is known. In order to take the stochastic uncertainty into account appropriately, deterministic formulations of the stochastic linear complementarity problem (SLCP)

$$
M(\omega) x+q(\omega) \geq 0, x \geq 0, x^{T}(M(\omega) x+q(\omega))=0, \quad \omega \in \Omega
$$

\footnotetext{
${ }^{1}$ This work was supported in part by a Grant-in-Aid for Scientific Research from Japan Society for the Promotion of Science.
} 
have been studied. We denote problem (1.1) by $\operatorname{SLCP}(M(\omega), q(\omega))$. In this paper, we consider two existing deterministic formulations. Let us denote

$$
y(x, \omega):=M(\omega) x+q(\omega) .
$$

Let $\phi: R^{2} \rightarrow R$ be a function, called an NCP function, which satisfies

$$
\phi(a, b)=0 \quad \Longleftrightarrow \quad a \geq 0, b \geq 0, a b=0 .
$$

Then it is easy to verify that for each $\omega \in \Omega, x_{\omega}$ is a solution of (1.1) if and only if it is an optimal solution of the following minimization problem with zero objective value:

$$
\min _{x \in R_{+}^{n}}\|\Phi(x, \omega)\|^{2}
$$

where $R_{+}^{n}:=\left\{x \in R^{n} \mid x \geq 0\right\}$ and

$$
\Phi(x, \omega):=\left(\begin{array}{c}
\phi\left(y_{1}(x, \omega), x_{1}\right) \\
\vdots \\
\phi\left(y_{n}(x, \omega), x_{n}\right)
\end{array}\right) .
$$

In the literature of linear complementarity problems, $\|\Phi(x, \omega)\|$ is called a residual for $\operatorname{LCP}(M(\omega), q(\omega))$, since $x_{\omega}$ solves $\operatorname{LCP}(M(\omega), q(\omega))$ if and only if it solves $\Phi(x, \omega)=0$. On the other hand, from the literature of stochastic optimization, $\|\Phi(x, \omega)\|^{2}$ can be regarded as a random cost function for $\operatorname{LCP}(M(\omega), q(\omega))$. In this sense, a deterministic formulation for the SLCP called the expected residual minimization problem in [3] may be regarded as an expected total cost minimization problem $[1,12,18]$ for $(1.1)$.

\section{- Expected Residual Minimization (ERM) Formulation [3]:}

Find a vector $x \in R_{+}^{n}$ that minimizes the expected total residual defined by an NCP function:

$$
\min _{x \in R_{+}^{n}} f(x):=E\left[\|\Phi(x, \omega)\|^{2}\right],
$$

where $E\left[\|\Phi(x, \omega)\|^{2}\right]$ is the expectation function of the random function $\|\Phi(x, \omega)\|^{2}$.

The expectation function of the random function $y(x, \omega)$ yields another deterministic formulation [11] for SLCP, which may be called the expected value formulation.

\section{- Expected Value (EV) Formulation [11]:}

Find a vector $x \in R^{n}$ such that

$$
\bar{y}(x):=E[y(x, \omega)] \geq 0, x \geq 0, x^{T} \bar{y}(x)=0 .
$$

Let

$$
\bar{M}=E[M(\omega)] \quad \text { and } \quad \bar{q}=E[q(\omega)]
$$

be the expectation matrix and vector of the random matrix $M(\cdot)$ and vector $q(\cdot)$, respectively. Then $\bar{y}(x)=\bar{M} x+\bar{q}$ and the EV formulation (1.5) is to find a solution of the $\operatorname{LCP}(\bar{M}, \bar{q})$. 
Both formulations (1.4) and (1.5) seek solutions in the nonnegative orthant. The nonnegative constraint $x \geq 0$ does not involve uncertain data and is required in numerous applications of engineering and economic [9]. Approximations of the expectation functions in (1.4) and (1.5) are investigated in [3, 11].

We call $M(\cdot)$ a stochastic $R_{0}$ matrix [7] if

$$
x \geq 0, M(\omega) x \geq 0, x^{T} M(\omega) x=0, \text { a.e. } \quad \Longrightarrow \quad x=0 .
$$

If $\Omega$ only contains a single element $\omega$, then $M(\omega)$ is an $R_{0}$ matrix. However, $M(\cdot)$ being a stochastic $R_{0}$ matrix does not imply that there is an $\omega \in \Omega$ such that $M(\omega)$ is an $R_{0}$ matrix. See Example 2.1 in [7].

Let $S_{E R M}$ and $S_{E V}$ be the solution sets of the ERM formulation (1.4) and EV formulation (1.5), respectively. In [7], the following results are shown: If $S_{E V}$ is bounded for any $\bar{q}$, then $S_{E R M}$ is bounded for any $q(\cdot)$, but the converse is not true in general. Moreover, the random matrix $M(\cdot)$ being a stochastic $R_{0}$ matrix is a necessary and sufficient condition for the solution set $S_{E R M}$ to be nonempty and bounded for any random vector $q(\cdot)$.

If the expectation matrix $\bar{M}$ is an $R_{0}$ matrix, then $M(\cdot)$ is a stochastic $R_{0}$ matrix; but the converse is not true. Since a positive definite matrix is an $R_{0}$ matrix, we can claim that if the expectation matrix $\bar{M}$ is a positive definite matrix, then the solution set $S_{E R M}$ is nonempty and bounded for any $q(\cdot)$. However, a positive semi-definite matrix may not be an $R_{0}$-matrix.

In this paper, we focus our attention on the SLCP (1.1) with the expectation matrix $\bar{M}$ being a positive semi-definite matrix, i.e.,

$$
x^{T} \bar{M} x \geq 0 \text { for all } x \in R^{n} .
$$

We call (1.1) a monotone $S L C P$ if $\bar{M}$ is a positive semi-definite matrix. Note that $\bar{M}$ being a positive semi-definite matrix does not imply that $M(\cdot)$ is a stochastic $R_{0}$ matrix. New analysis on the solution set $S_{E R M}$ has to be studied for monotone SLCPs.

Obviously, if $M(\omega)$ is a positive semi-definite matrix for all $\omega \in \Omega$, then $\bar{M}$ is a positive semi-definite matrix. However, the expectation matrix $\bar{M}$ being a positive semi-definite matrix does not imply that

$$
\mathcal{P}\{\omega \in \Omega \mid M(\omega) \text { is positive semi-definite }\}>0 .
$$

In the following example, $\bar{M}$ is a positive definite matrix, i.e.,

$$
x^{T} \bar{M} x>0 \text { for all nonzero } x \in R^{n},
$$

but there is no $\omega \in \Omega$ such that $M(\omega)$ is a positive semi-definite matrix.

Example 1.1 Let

$$
M(\omega)=\left(\begin{array}{cc}
-5+(15+\omega) \max (0, \operatorname{sign}(\omega)) & 0 \\
0 & -5-(15+\omega) \min (0, \operatorname{sign}(\omega))
\end{array}\right),
$$


where $\omega \in \Omega=[-1,1]$ and $\omega$ is uniformly distributed on $\Omega$. It is easy to see that

$$
\begin{gathered}
M(\omega)=\left(\begin{array}{cc}
-5 & 0 \\
0 & 10+\omega
\end{array}\right) \quad \text { for } \omega<0, \quad M(\omega)=\left(\begin{array}{cc}
10+\omega & 0 \\
0 & -5
\end{array}\right) \text { for } \omega>0, \\
M(\omega)=\left(\begin{array}{cc}
-5 & 0 \\
0 & -5
\end{array}\right) \quad \text { for } \omega=0, \quad \bar{M}=E(M(\omega))=\left(\begin{array}{cc}
2.75 & 0 \\
0 & 2.25
\end{array}\right) .
\end{gathered}
$$

Although the positive definiteness of $\bar{M}$ does not ensure the existence of an $\omega \in \Omega$ such that $M(\omega)$ is positive semi-definite, we find that the monotone $\operatorname{LCP}(\bar{M}, \bar{q})$ serves as an important tool in the study of the monotone SLCP with the ERM formulation. In particular, we will show that if the monotone $\operatorname{LCP}(\bar{M}, \bar{q})$ has a bounded solution set $S_{E V}$, then the ERM formulation (1.4) of the monotone SLCP has a bounded solution set

$S_{E R M}$. Without any assumption on the solution set $S_{E V}$, we will prove that $\bar{M}$ being positive semi-definite implies that every accumulation point of a sequence generated by the regularization method is a solution of the ERM formulation (1.4).

In general, the two deterministic formulations (1.4) and (1.5) have different solutions. Moreover, with different NCP functions and norms, the ERM formulation has different solutions. How to select a robust solution that is insensitive with respect to random parameter variations is an important issue in decision theory. To investigate the characteristics of optimal solutions of the ERM formulation, we give a new error bound for the monotone LCP based on the error bounds in [17]. Using the error bound, we will show that optimal solutions of the ERM formulation (1.4) yield a high mean performance of the SLCP and may have a minimum sensitivity with respect to random parameter variations in SLCP. Hence, they are robust solutions for SLCP.

This paper is organized as follows: In Section 2, we study the existence of solutions for the ERM formulation of the monotone SLCP based on the monotone $\operatorname{LCP}(\bar{M}, \bar{q})$. In Section 3, we investigate the robustness of the ERM formulation. In Section 4, we give a procedure to generate a test problem of monotone SLCP, which allows the user to specify the size of the problem, the condition number of the expectation matrix $\bar{M}$ and the number of active constraints at a global solution of the ERM formulation. We report numerical results for hundreds of test problems by using a semismooth Newtontype method with a descent direction line search. In Section 5, we describe a realistic application, traffic equilibrium under uncertainty.

In this paper, $\|\cdot\|$ denotes the Euclidean norm $\|\cdot\|_{2}$. For any positive integer $s$ and a vector $z \in R^{s}$, we denote $[z]_{+}=\max (0, z)$, where the maximum is taken component-wise. For a subset $J \subseteq\{1,2, \ldots, s\}, z_{J}$ denotes the subvector of $z$ with components $z_{j}, j \in J$. For a matrix $A$, let $A_{i}$ represent the $i$ th row of $A$. Let $\mathcal{N}=\{1,2, \ldots, N\}$.

\section{Existence of solution}

In this section, we study the relation between the $\mathrm{EV}$ formulation $\operatorname{LCP}(\bar{M}, \bar{q})$ and the ERM formulation of the monotone SLCP. First, we summarize some results on the exis- 
tence of a solution for a deterministic monotone LCP. Recall that a square matrix $A$ is called an $R_{0}$ matrix if the solution set of $\operatorname{LCP}(A, 0)$ consists of the origin only.

Lemma 2.1 Suppose that $A$ is a positive semi-definite matrix.

(i) [4] If the $\operatorname{LCP}(A, p)$ is feasible, i.e., there is a vector $x \geq 0$ such that $A x+p \geq 0$, then it has a solution.

(ii) [4] The $L C P(A, p)$ has a nonempty and bounded solution set for any $p$ if and only if $A$ is in addition an $R_{0}$ matrix.

(iii) [2] The solution set of $\operatorname{LCP}(A, p)$ is nonempty and bounded if and only if $L C P(A, p)$ has a strictly feasible point, i.e., there is a vector $x>0$ such that $A x+p>0$.

The ERM formulation (1.4) utilizes an NCP function that possesses the property (1.2). There are a variety of functions that satisfy (1.2). Among them, the most popular NCP functions are the "min" function $\phi_{1}$ and the Fischer-Burmeister (FB) function $\phi_{2}$, which are defined by

$$
\phi_{1}(a, b):=\min (a, b)
$$

and

$$
\phi_{2}(a, b):=a+b-\sqrt{a^{2}+b^{2}},
$$

respectively. Notice that, as shown below, the solvability of the ERM formulation is dependent on the choice of NCP functions.

Example 2.1 [3] Let $n=1, m=1, \Omega=\left\{\omega^{1}, \omega^{2}\right\}=\{0,1\}, M(\omega)=\omega(1-\omega)$ and $q(\omega)=1-2 \omega, M\left(\omega^{1}\right)=M\left(\omega^{2}\right)=0, q\left(\omega^{1}\right)=1, q\left(\omega^{2}\right)=-1$ and

$$
E\left[\|\Phi(x, \omega)\|^{2}\right]=\frac{1}{2} \sum_{i=1}^{2}\left\|\Phi\left(x, \omega^{i}\right)\right\|^{2} .
$$

For every $\omega \in \Omega, M(\omega)$ is positive semi-definite. It can be seen that the ERM problem (1.4) defined by the "min" function has the unique solution $x^{*}=0$ and the level set

$$
\left\{x \mid E\left[\|\min (x, M(\omega) x+q(\omega))\|^{2}\right] \leq \gamma\right\}
$$

is nonempty and bounded for all $\gamma \in[0.5,1)$. However, the ERM problem (1.4) defined by the FB function does not have a solution as the objective function is monotonically decreasing on $[0, \infty)$.

Nevertheless, the FB function has a number of nice properties. Among others, a distinctive property from the "min" function is that $\|\Phi(\cdot, \omega)\|^{2}$ defined by the FB function is continuously differentiable everywhere. However, the FB function lacks flexibility in dealing with the monotone LCP. Some other merit functions and NCP functions have nice properties in dealing with monotone LCP $[2,13,16,20]$. Here, we consider a version of the penalized FB NCP function given in [2]

$$
\phi_{3}(a, b):=\lambda\left(a+b-\sqrt{a^{2}+b^{2}}\right)+(1-\lambda) a_{+} b_{+},
$$


where $\lambda \in(0,1)$. For Example 2.1, the ERM formulation (1.4) defined by $\phi_{3}$ with $\lambda=\frac{1}{2}$ has the objective function

$$
f_{3}(x)=\frac{1}{4}\left[\left(1+x-\sqrt{1+x^{2}}+x_{+}\right)^{2}+\left(-1+x-\sqrt{1+x^{2}}\right)^{2}\right],
$$

which is a continuously differentiable convex function and has a minimizer $x^{*} \approx 0.3685$. Moreover, the level set $\left\{x \mid f_{3}(x) \leq \gamma\right\}$ is nonempty and bounded for all $\gamma \in\left[f_{3}\left(x^{*}\right), \infty\right)$.

The NCP functions $\phi_{1}$ and $\phi_{2}$ have the same growth rate. In particular, Tseng [19] showed

$$
\frac{2}{\sqrt{2}+2}|\min (a, b)| \leq\left|a+b-\sqrt{a^{2}+b^{2}}\right| \leq(\sqrt{2}+2)|\min (a, b)| \quad \text { for } \quad(a, b) \in R^{2} .
$$

However, for $\phi_{1}$ and $\phi_{3}$, we only have

$$
\min (\lambda, 1-\lambda) \frac{2}{\sqrt{2}+2}|\min (a, b)| \leq\left|\lambda\left(a+b-\sqrt{a^{2}+b^{2}}\right)+(1-\lambda) a_{+} b_{+}\right| \quad \text { for } \quad(a, b) \in R^{2} .
$$

There is no $c>0$ such that

$$
c|\min (a, b)| \geq\left|\lambda\left(a+b-\sqrt{a^{2}+b^{2}}\right)+(1-\lambda) a_{+} b_{+}\right| \quad \text { for } \quad(a, b) \in R^{2} .
$$

The ERM formulation (1.4) defined by the "min" function and the penalized FB function has different properties in regard to smoothness and boundedness. When we discuss their different properties, we use $\Phi_{1}(x, \omega), f_{1}(x)$, and $\Phi_{3}(x, \omega), f_{3}(x)$ to distinguish the functions $\Phi(x)$ and $f(x)$ defined by the "min" function $\phi_{1}$ and the penalized FB function $\phi_{3}$, respectively. When we discuss the ERM formulation (1.4) defined by any of the NCP functions, we use the notation $\Phi(x, \omega)$ and $f(x)$.

Assumption I. $f(x)$ is finite and continuous at any $x \in R_{+}^{n}$.

This assumption holds if $M(\omega)$ and $q(\omega)$ are measurable functions of $\omega$ with the following property

$$
E\left[(\|M(\omega)\|+\|q(\omega)\|)^{2}\right]<\infty .
$$

Let us denote the expected value of random function $\Phi(\cdot, x)$ by

$$
\bar{\Phi}(x):=E[\Phi(x, \omega)]
$$

By probability theory, we have Jensen's inequality for the objective function $f$

$$
E\left[\|\Phi(x, \omega)\|^{2}\right] \geq\|E[\Phi(x, \omega)]\|^{2}=\|\bar{\Phi}(x)\|^{2} .
$$

\section{1 "min" function}

In this subsection, we consider the ERM formulation (1.4) defined by the "min" function.

Lemma 2.2 If $\Omega=\left\{\omega^{1}, \omega^{2}, \ldots, \omega^{N}\right\}$, then for any random matrix $M(\cdot)$ and vector $q(\cdot)$, the solution set $S_{E R M}$ of the ERM formulation (1.4) defined by the "min" function is nonempty. 
Proof: For each $\omega^{\nu}$, the squared norm of the function $\Phi_{1}\left(x, \omega^{\nu}\right)=\min \left(x, M\left(\omega^{\nu}\right) x+\right.$ $\left.q\left(\omega^{\nu}\right)\right)$ can be represented as

$$
\left\|\Phi_{1}\left(x, \omega^{\nu}\right)\right\|^{2}=\left(M\left(\omega^{\nu}\right)^{j} x+q\left(\omega^{\nu}\right)^{j}\right)^{T}\left(M\left(\omega^{\nu}\right)^{j} x+q\left(\omega^{\nu}\right)^{j}\right), \quad x \in P_{\nu}^{j}, \quad j=1, \ldots, k,
$$

where $P_{\nu}^{j}$ are polyhedral convex sets comprising a partition of $R_{+}^{n}$, each $\left(M\left(\omega^{\nu}\right)^{j}, q\left(\omega^{\nu}\right)^{j}\right)$ is a row representative of $\left(\left(I, M\left(\omega^{\nu}\right)\right),\left(0, q\left(\omega^{\nu}\right)\right)\right)$, and $k \leq 2^{n}$. Hence $f_{1}$ is a piecewise quadratic function and $f_{1}(x) \geq 0$ on $R_{+}^{n}$. By the Frank-Wolfe Theorem [10], $f_{1}$ attains its minimum on $R_{+}^{n}$.

From the proof of Lemma 2.2, if $\Omega=\left\{\omega^{1}, \omega^{2}, \ldots, \omega^{N}\right\}$, then $f_{1}$ is a piecewise quadratic function. However, the following example shows that for a continuous random variable, $f_{1}$ is not necessarily a piecewise quadratic function.

Example 2.2 Let $n=1, m=1, M(\omega)=1+\omega, q(\omega) \equiv-1, \omega \in \Omega=[0,1]$, where $\omega$ is uniformly distributed on $\Omega$. By direct calculation, we find

$$
f_{1}(x)=E|\min (x,(1+\omega) x-1)|^{2}= \begin{cases}\frac{1}{3}\left(7 x^{2}-9 x+3\right), & 0 \leq x \leq 1 \\ x^{2}+\frac{1}{3 x}-1, & x>1 .\end{cases}
$$

Denote the level set

$$
D_{1}(\gamma):=\left\{x \mid f_{1}(x) \leq \gamma\right\}
$$

$D_{1}(\gamma)$ may be empty for some $\gamma>0$, since the minimum value of $f_{1}(x)$ is positive in general.

Theorem 2.1 Assume that $\bar{M}$ is a positive semi-definite matrix. If there are $\hat{x}, \bar{x} \in R^{n}$ such that

$$
\min _{1 \leq i \leq n}(\bar{M} \hat{x}+\bar{q})_{i}>\sqrt{f_{1}(\bar{x})}=: \bar{\gamma}
$$

and there exists a vector $d>0$ such that $d^{T} \bar{M} x$ is bounded above in $D_{1}\left(\bar{\gamma}^{2}\right)$, then the level set $D_{1}\left(\bar{\gamma}^{2}\right)$ is nonempty and bounded.

Proof: Since $f_{1}(\bar{x})=\bar{\gamma}^{2}$, the level set $D_{1}\left(\bar{\gamma}^{2}\right)$ is nonempty. Suppose on the contrary that there exists an unbounded sequence $\left\{x^{k}\right\} \subset D_{1}\left(\bar{\gamma}^{2}\right)$. By Jensen's inequality (2.5), we find

$$
\left\|E\left[\min \left(x^{k}, y\left(x^{k}, \omega\right)\right)\right]\right\|=\left\|E\left[\Phi_{1}\left(x^{k}, \omega\right)\right]\right\| \leq \sqrt{E\left[\left\|\Phi_{1}\left(x^{k}, \omega\right)\right\|^{2}\right]}=\sqrt{f_{1}\left(x^{k}\right)} \leq \bar{\gamma} .
$$

Hence, it is clear that for any index $i$, both $\left\{x_{i}^{k}\right\}$ and $\left\{\bar{y}_{i}\left(x^{k}\right)\right\}$ are bounded below. Since $\bar{y}_{i}\left(x^{k}\right)=\left(\bar{M} x^{k}+\bar{q}\right)_{i},\left\{\left(\bar{M} x^{k}\right)_{i}\right\}$ is also bounded below for all $i \in \mathcal{N}$. Moreover, by assumption, $\left\{d^{T} \bar{M} x^{k}\right\}$ is bounded above. Therefore, we can conclude that for each $i$, $\left\{\bar{y}_{i}\left(x^{k}\right)\right\}$ is bounded above. Define the index set

$$
J=\left\{i \mid \limsup _{k \rightarrow \infty} x_{i}^{k}=\infty\right\}
$$

Note that this set is not empty. By taking a subsequence if necessary, we may suppose that $x_{i}^{k} \rightarrow \infty$ for $i \in J$. Then we have for all sufficiently large $k$

$$
\left|E\left[\min \left(x_{i}^{k}, y_{i}\left(x^{k}, \omega\right)\right)\right]\right|=\left|E\left[y_{i}\left(x^{k}, \omega\right)\right]\right| \leq \bar{\gamma} \quad \text { for } \quad i \in J,
$$


which together with (2.6) yields

$$
E\left[y_{i}\left(x^{k}, \omega\right)\right] \leq \bar{\gamma}<(\bar{M} \hat{x}+\bar{q})_{i}=E\left[y_{i}(\hat{x}, \omega)\right] \quad \text { for } \quad i \in J
$$

that is,

$$
\bar{y}_{i}\left(x^{k}\right)-\bar{y}_{i}(\hat{x})=E\left[y_{i}\left(x^{k}, \omega\right)-y_{i}(\hat{x}, \omega)\right] \leq \bar{\gamma}-E\left[y_{i}(\hat{x}, \omega)\right]<0 \quad \text { for } \quad i \in J .
$$

Moreover, $\left\{x_{i}^{k}\right\}$ and $\left\{\bar{y}_{i}\left(x^{k}\right)\right\}$ are bounded for each $i \notin J$. Therefore, we have

$$
\begin{aligned}
\left(x^{k}-\hat{x}\right)^{T} \bar{M}\left(x^{k}-\hat{x}\right) & =\left(x^{k}-\hat{x}\right)^{T} E\left[y\left(x^{k}, \omega\right)-y(\hat{x}, \omega)\right] \\
& =\sum_{i \in J}\left(x_{i}^{k}-\hat{x}_{i}\right)\left(\bar{y}_{i}\left(x^{k}\right)-\bar{y}_{i}(\hat{x})\right)+\sum_{i \notin J}\left(x_{i}^{k}-\hat{x}_{i}\right)\left(\bar{y}_{i}\left(x^{k}\right)-\bar{y}_{i}(\hat{x})\right) .
\end{aligned}
$$

The first term tends to $-\infty$ while the second term is bounded. This contradicts the positive semi-definiteness of $\bar{M}$. Hence $D_{1}\left(\bar{\gamma}^{2}\right)$ is bounded.

Corollary 2.1 Under the assumptions of Theorem 2.1 with $\bar{x} \geq 0$, the solution set $S_{E R M}$ of the ERM formulation (1.4) defined by the "min" function is nonempty and bounded.

Remark 2.1 If $\bar{M}$ is positive definite, then $\bar{M}$ is an $R_{0}$ matrix. From Proposition 2.5 and Theorem $3.1 \mathrm{in} \mathrm{[7],} M(\cdot)$ is a stochastic $R_{0}$ matrix, and the level set $D_{1}(\gamma)$ is bounded for any $\gamma>0$. Even if $\bar{M}$ is not positive definite, the level set $D_{1}\left(\bar{\gamma}^{2}\right)$ is bounded, particularly when there is a vector $d>0$ such that $\bar{M}^{T} d=0$ and (2.6) holds. In fact, it is easy to see that these conditions guarantee the assumptions in Theorem 2.1.

The following example shows that the assumptions in Theorem 2.1 hold with $\bar{x} \geq 0$, but the monotone $\operatorname{LCP}(\bar{M}, \bar{q})$ does not have a solution.

Example 2.3 Let $n=2, m=2, \Omega=\left\{\omega^{1}, \omega^{2}\right\} \subset R^{2}, \omega^{1}=(0,1), \omega^{2}=(1,0)$,

$$
M(\omega)=\left(\begin{array}{cc}
0 & -\omega_{1} \\
\omega_{1} & \omega_{1}
\end{array}\right), \quad q(\omega)=\left(\begin{array}{c}
-2 \omega_{1}+\omega_{2} \\
\omega_{1}+\omega_{2}
\end{array}\right),
$$

and

$$
E\left[\|\Phi(x, \omega)\|^{2}\right]=\frac{1}{2} \sum_{i=1}^{2}\left\|\Phi\left(x, \omega^{i}\right)\right\|^{2} .
$$

Then we have

$$
M\left(\omega^{1}\right)=\left(\begin{array}{ll}
0 & 0 \\
0 & 0
\end{array}\right), M\left(\omega^{2}\right)=\left(\begin{array}{cc}
0 & -1 \\
1 & 1
\end{array}\right), q\left(\omega^{1}\right)=\left(\begin{array}{l}
1 \\
1
\end{array}\right), q\left(\omega^{2}\right)=\left(\begin{array}{c}
-2 \\
1
\end{array}\right),
$$

and

$$
\bar{M}=\left(\begin{array}{cc}
0 & -1 / 2 \\
1 / 2 & 1 / 2
\end{array}\right), \quad \bar{q}=\left(\begin{array}{c}
-1 / 2 \\
1
\end{array}\right)
$$


Obviously, $M(\omega)$ is positive semi-definite for each $\omega \in \Omega$. For $x \in R_{+}^{2}$, we have

$$
\begin{aligned}
f_{1}(x)= & \frac{1}{2}\left[\left|\min \left(x_{1}, 1\right)\right|^{2}+\left|\min \left(x_{2}, 1\right)\right|^{2}\right. \\
& \left.+\left|\min \left(x_{1},-x_{2}-2\right)\right|^{2}+\left|\min \left(x_{2}, x_{1}+x_{2}+1\right)\right|^{2}\right] \\
= & \frac{1}{2}\left[\left|\min \left(x_{1}, 1\right)\right|^{2}+\left|\min \left(x_{2}, 1\right)\right|^{2}+\left(x_{2}+2\right)^{2}+x_{2}^{2}\right] .
\end{aligned}
$$

By direct calculation, we see that the ERM formulation $\min _{x \in R_{+}^{2}} f_{1}(x)$ has $x=0$ as its unique solution with optimal value $f_{1}(x)=2$. Moreover, it is easy to verify that the level set $D_{1}(\gamma)$ is bounded for any $\gamma \in[2,2.5)$ and assumptions in Theorem 2.1 hold with $\hat{x}=(9,-6)^{T}, \bar{x}=\left(\frac{1}{2}, 0\right)^{T}, d=(1,1)^{T}$, and $\bar{\gamma}=\sqrt{2.125}$. However, the $E V$ formulation $\operatorname{LCP}(\bar{M}, \bar{q})$ has no feasible point, since the first component of $\bar{M} x+\bar{q}$ is negative for any $x \in R_{+}^{2}$.

\subsection{Penalized FB function}

In this subsection, we consider the ERM formulation (1.4) with the penalized FB function $\phi_{3}$ defined by (2.1). Since the analysis remains valid for any $\lambda \in(0,1)$, in the definition of $\phi_{3}$, we omit $\lambda$ in the following discussion for simplicity of presentation.

Lemma 2.3 Let $\left\{x^{k}\right\}$ be an arbitrary sequence contained in the level set

$$
D_{3}(\gamma):=\left\{x \mid f_{3}(x) \leq \gamma\right\}
$$

Then $\left\{x_{i}^{k}\right\}$ and $\left\{E\left[y_{i}\left(x^{k}, \omega\right) 1_{\left\{\omega \in \Omega_{0}\right\}}\right]\right\}$ are bounded below, and $\left\{x_{i}^{k} E\left[y_{i}\left(x^{k}, \omega\right) 1_{\left\{\omega \in \Omega_{0}\right\}}\right]\right\}$ is bounded above for any index $i \in \mathcal{N}$ and any subset $\Omega_{0} \subseteq \Omega$.

Proof: First we show that $\left\{x_{i}^{k}\right\}$ and $\left\{E\left[y_{i}\left(x^{k}, \omega\right)\right]\right\}$ are bounded below for all $i \in \mathcal{N}$. By (2.3) and Jensen's inequality (2.5), we have

$$
\begin{aligned}
f_{3}(x) & =\sum_{i=1}^{n} E\left[\left(\phi_{3}\left(x_{i}, y_{i}(x, \omega)\right)\right)^{2}\right] \\
& \geq \frac{4}{(\sqrt{2}+2)^{2}} \sum_{i=1}^{n} E\left[\left(\phi_{1}\left(x_{i}, y_{i}(x, \omega)\right)\right)^{2}\right] \\
& \geq \frac{4}{(\sqrt{2}+2)^{2}} \sum_{i=1}^{n}\left(E\left[\phi_{1}\left(x_{i}, y_{i}(x, \omega)\right)\right]\right)^{2} \\
& =\frac{4}{(\sqrt{2}+2)^{2}}\left\|E\left[\Phi_{1}(x, \omega)\right]\right\|^{2} .
\end{aligned}
$$

Moreover, it is easy to verify that for each $i$

$$
E\left[\min \left(x_{i}, y_{i}(x, \omega)\right)\right] \leq \min \left(x_{i}, E\left[y_{i}(x, \omega)\right]\right),
$$

which implies that $E\left[\min \left(x_{i}^{k}, y_{i}\left(x^{k}, \omega\right)\right)\right] \rightarrow-\infty$ if $x_{i}^{k} \rightarrow-\infty$ or $E\left[y_{i}\left(x^{k}, \omega\right)\right] \rightarrow-\infty$. Hence there is no index $i$ such that $x_{i}^{k} \rightarrow-\infty$ or $\bar{y}_{i}\left(x^{k}\right)=E\left[y_{i}\left(x^{k}, \omega\right)\right] \rightarrow-\infty$. 
Next, we show that the sequence $\left\{x_{i}^{k} E\left[y_{i}\left(x^{k}, \omega\right)\right]\right\}$ is bounded above. If it is not true, we may suppose without loss of generality that $x_{j}^{k} E\left[y_{j}\left(x^{k}, \omega\right)\right] \rightarrow \infty$ for some index $j$. Then there are two cases: (i) There is a subsequence $\left\{x_{j}^{k_{i}}\right\}$ such that $x_{j}^{k_{i}} \rightarrow \infty$, and (ii) $\left\{x_{j}^{k}\right\}$ is positive and bounded.

(i) Suppose there is a subsequence such that $x_{j}^{k_{i}} \rightarrow \infty$. Since

$$
0<E\left[y_{j}\left(x^{k_{i}}, \omega\right)\right]=E\left[\left[y_{j}\left(x^{k_{i}}, \omega\right)\right]_{+}\right]-E\left[\left[-y_{j}\left(x^{k_{i}}, \omega\right)\right]_{+}\right]
$$

we find

$$
E\left[\left[-y_{j}\left(x^{k_{i}}, \omega\right)\right]_{+}\right]<E\left[\left[y_{j}\left(x^{k_{i}}, \omega\right)\right]_{+}\right]
$$

and

$$
\begin{aligned}
E\left[\sqrt{\left(x_{j}^{k_{i}}\right)^{2}+y_{j}\left(x^{k_{i}}, \omega\right)^{2}}\right] & \leq E\left[\left|x_{j}^{k_{i}}\right|+\left|y_{j}\left(x^{k_{i}}, \omega\right)\right|\right] \\
& =x_{j}^{k_{i}}+E\left[\left[y_{j}\left(x^{k_{i}}, \omega\right)\right]_{+}\right]+E\left[\left[-y_{j}\left(x^{k_{i}}, \omega\right)\right]_{+}\right]
\end{aligned}
$$

for all $k_{i}$ large enough. Therefore, as $x_{j}^{k_{i}} E\left[y_{j}\left(x^{k_{i}}, \omega\right)\right] \rightarrow \infty$,

$$
\begin{aligned}
f_{3}\left(x^{k_{i}}\right) & =\left\|E\left[\Phi_{3}\left(x^{k_{i}}, \omega\right)\right]\right\|^{2} \\
& \geq\left|E\left[\Phi_{3}\left(x^{k_{i}}, \omega\right)\right]_{j}\right|^{2} \\
& =\left|x_{j}^{k_{i}}+E\left[y_{j}\left(x^{k_{i}}, \omega\right)\right]-E\left[\sqrt{\left(x_{j}^{k_{i}}\right)^{2}+y_{j}\left(x^{k_{i}}, \omega\right)^{2}}\right]+\left[x_{j}^{k_{i}}\right]_{+} E\left[\left[y_{j}\left(x^{k_{i}}, \omega\right)\right]_{+}\right]\right|^{2} \\
& \geq\left|-2 E\left[\left[-y_{j}\left(x^{k_{i}}, \omega\right)\right]_{+}\right]+\left[x_{j}^{k_{i}}\right]_{+} E\left[\left[y_{j}\left(x^{k_{i}}, \omega\right)\right]_{+}\right]\right|^{2} \\
& \geq\left(\left(x_{j}^{k_{i}}-2\right)\left[\bar{y}_{j}\left(x^{k_{i}}\right)\right]_{+}\right)^{2} \\
& \rightarrow \infty
\end{aligned}
$$

where the second inequality uses (2.7) and (2.9), and the third inequality uses (2.8) and $E\left[\xi_{+}\right] \geq(E[\xi])_{+}$for any random variable $\xi$. This contradicts $\left\{x^{k}\right\} \subset D_{3}(\gamma)$.

(ii) Suppose $\left\{x_{j}^{k}\right\}$ is positive and bounded. Then we have $E\left[y_{j}\left(x^{k}, \omega\right)\right] \rightarrow \infty$. There are a subset $\hat{\Omega} \subseteq \Omega$ and a vector $x^{\hat{k}} \in\left\{x^{k}\right\}$ such that

$$
y_{j}\left(x^{\hat{k}}, \omega\right) \geq 0 \quad \forall \omega \in \hat{\Omega} \quad \text { and } \quad x_{j}^{\hat{k}} E\left[y_{j}\left(x^{\hat{k}}, \omega\right) 1_{\{\omega \in \hat{\Omega}\}}\right]>\sqrt{\gamma} .
$$

The former condition yields

$$
x_{j}^{\hat{k}}+y_{j}\left(x^{\hat{k}}, \omega\right)-\sqrt{\left(x_{j}^{\hat{k}}\right)^{2}+y_{j}\left(x^{\hat{k}}, \omega\right)^{2}} \geq 0 \quad \text { for all } \omega \in \hat{\Omega} .
$$

Hence, we find

$$
\begin{aligned}
f_{3}\left(x^{\hat{k}}\right) & \geq E\left[\left|\left(\Phi_{3}\left(x^{\hat{k}}, \omega\right)\right)_{j}\right|^{2} 1_{\{\omega \in \hat{\Omega}\}}\right] \\
& =E\left[\left|x_{j}^{\hat{k}}+y_{j}\left(x^{\hat{k}}, \omega\right)-\sqrt{\left(x_{j}^{\hat{k}}\right)^{2}+y_{j}\left(x^{\hat{k}}, \omega\right)^{2}}+\left[x_{j}^{\hat{k}}\right]_{+}\left[y_{j}\left(x^{\hat{k}}, \omega\right)\right]_{+}\right|^{2} 1_{\{\omega \in \hat{\Omega}\}}\right] \\
& \geq\left(x_{j}^{\hat{k}} E\left[y_{j}\left(x^{\hat{k}}, \omega\right) 1_{\{\omega \in \hat{\Omega}\}}\right]\right)^{2} \\
& >\gamma .
\end{aligned}
$$


This contradicts $x^{\hat{k}} \in\left\{x^{k}\right\} \subset D_{3}(\gamma)$.

We have proved that $\left\{x^{k}\right\}$ and $\left\{E\left[y_{i}\left(x^{k}, \omega\right)\right]\right\}$ are bounded below, and the sequence $\left\{x_{i}^{k} E\left[y_{i}\left(x^{k}, \omega\right)\right]\right\}$ is bounded above for any index $i$. By noticing that for any subset $\Omega_{0} \subseteq \Omega$,

$$
E\left[\left\|\Phi(x, \omega) 1_{\left\{\omega \in \Omega_{0}\right\}}\right\|\right] \leq E[\|\Phi(x, \omega)\|],
$$

and following the above analysis, we can obtain without difficulty that for any sequence $\left\{x^{k}\right\} \subset D_{3}(\gamma), i \in \mathcal{N}$, and $\Omega_{0} \subseteq \Omega$, the sequence $\left\{E\left[y_{i}\left(x^{k}, \omega\right) 1_{\left\{\omega \in \Omega_{0}\right\}}\right]\right\}$ is bounded below, and the sequence $\left\{x_{i}^{k} E\left[y_{i}\left(x^{k}, \omega\right) 1_{\left\{\omega \in \Omega_{0}\right\}}\right]\right\}$ is bounded above.

Let us denote the feasible set of $\operatorname{LCP}(\bar{M}, \bar{q})$ by $\mathcal{F}$. Suppose $\mathcal{F} \neq \emptyset$, and define

$$
\bar{\alpha}:=\left\{i:(\bar{M} x+\bar{q})_{i}=0 \text { for all } x \in \mathcal{F}\right\} .
$$

It is worth noting that the monotone $\operatorname{LCP}(\bar{M}, \bar{q})$ has a nonempty and bounded solution set $S_{E V}$ if and only if $\bar{\alpha}=\emptyset$. In fact, by Lemma 2.1, if $S_{E V}$ is bounded, then there exists a strictly feasible point, which implies $\bar{\alpha}=\emptyset$. If $\bar{\alpha}=\emptyset$, but there is a sequence $\left\{x^{k}\right\} \subseteq S_{E V}$ with $x_{i}^{k} \rightarrow \infty$ for some $i$, then from $\bar{\alpha}=\emptyset$, there is $\tilde{x} \in \mathcal{F}$ such that $(\bar{M} \tilde{x}+\bar{q})_{i}>0$, which yields

$$
\left(x^{k}-\tilde{x}\right)^{T} \bar{M}\left(x^{k}-\tilde{x}\right)=-\left(x^{k}\right)^{T}(\bar{M} \tilde{x}+\bar{q})-\tilde{x}^{T}\left(\bar{M} x^{k}+\bar{q}\right)+\tilde{x}^{T}(\bar{M} \tilde{x}+\bar{q}) \rightarrow-\infty .
$$

This is a contradiction to the positive semi-definiteness of $\bar{M}$.

Theorem 2.2 Assume that the monotone $L C P(\bar{M}, \bar{q})$ has a solution, and either $\bar{\alpha}=\emptyset$ or for any index $i \in \bar{\alpha}$, there is no vector $x \in R_{+}^{n}$ such that

$$
(M(\omega) x+q(\omega))_{i}=0, \quad \text { a.e. } \quad \omega \in \Omega .
$$

Then for any $\gamma \geq 0$, the level set

$$
\hat{D}_{3}(\gamma):=\left\{x \in R_{+}^{n} \mid f_{3}(x) \leq \gamma\right\}
$$

is bounded.

Proof: For a fixed $\gamma>0$, we assume on the contrary that $\left\|x^{k}\right\| \rightarrow \infty$ and $\left\{x^{k}\right\} \subset \hat{D}_{3}(\gamma)$. From Lemma 2.3, the sequence $\left\{E\left[y_{i}\left(x^{k}, \omega\right) 1_{\left\{\omega \in \Omega_{0}\right\}}\right]\right\}$ is bounded below and the sequence $\left\{x_{i}^{k} E\left[y_{i}\left(x^{k}, \omega\right) 1_{\left\{\omega \in \Omega_{0}\right\}}\right]\right\}$ is bounded above for any index $i \in \mathcal{N}$ and any subset $\Omega_{0} \subseteq \Omega$.

Define the index set $J=\left\{i \mid x_{i}^{k} \rightarrow \infty\right\}$ and let $\hat{x} \in \mathcal{F}$ be arbitrarily chosen. From the positive semi-definiteness of $\bar{M}$, we have that for any $k$,

$$
\left(x^{k}-\hat{x}\right)^{T}\left[\bar{y}\left(x^{k}\right)-\bar{y}(\hat{x})\right]=\left(x^{k}-\hat{x}\right)^{T} \bar{M}\left(x^{k}-\hat{x}\right) \geq 0 .
$$

Then we have $\bar{y}_{J}(\hat{x})=(\bar{M} \hat{x}+\bar{q})_{J}=0$. In fact, since $\left\{\left(x^{k}\right)^{T} \bar{y}\left(x^{k}\right)\right\}$ and $\left\{-\hat{x}^{T} \bar{y}\left(x^{k}\right)\right\}$ are bounded above, and $-x_{i}^{k} \bar{y}_{i}(\hat{x}) \leq 0$ for any $i \in \mathcal{N}$, the existence of an index $i \in J$ such that $\bar{y}_{i}(\hat{x})>0$ would imply

$$
\begin{aligned}
\left(x^{k}-\hat{x}\right)^{T}\left[\bar{y}\left(x^{k}\right)-\bar{y}(\hat{x})\right] & =\hat{x}^{T} \bar{y}(\hat{x})+\left(x^{k}\right)^{T} \bar{y}\left(x^{k}\right)-\left(x^{k}\right)^{T} \bar{y}(\hat{x})-\hat{x}^{T} \bar{y}\left(x^{k}\right) \\
& \rightarrow-\infty
\end{aligned}
$$


which contradicts (2.11). From the arbitrariness of $\hat{x} \in \mathcal{F}$, we must have $J \subseteq \bar{\alpha}$. Since $J \neq \emptyset$, the index set $\bar{\alpha}$ is also nonempty.

Moreover, we can claim that the sequence $\left\{x_{i}^{k} E\left[y_{i}\left(x^{k}, \omega\right) 1_{\left\{\omega \in \Omega_{0}\right\}}\right]\right\}$ is also bounded below for any index $i \in \mathcal{N}$ and any subset $\Omega_{0} \subseteq \Omega$. Indeed, since $\left\{x_{i}^{k} E\left[y_{i}\left(x^{k}, \omega\right) 1_{\left\{\omega \in \Omega_{0}\right\}}\right]\right\}$ is bounded above for any $i \in \mathcal{N}$ and any $\Omega_{0} \subseteq \Omega$, the existence of an index $\hat{i} \in \mathcal{N}$ and a subset $\hat{\Omega} \subseteq \Omega$ such that $x_{\hat{i}}^{k} E\left[y_{\hat{i}}\left(x^{k}, \omega\right) 1_{\{\omega \in \hat{\Omega}\}}\right] \rightarrow-\infty$ will imply $\left(x^{k}\right)^{T} \bar{y}\left(x^{k}\right) \rightarrow-\infty$. However, since $\left\{\bar{y}\left(x^{k}\right)\right\}$ is bounded below, we also obtain (2.12), which again contradicts (2.11).

Since the sequence $\left\{x_{i}^{k} E\left[y_{i}\left(x^{k}, \omega\right) 1_{\left\{\omega \in \Omega_{0}\right\}}\right]\right\}$ is bounded for any index $i \in \mathcal{N}$ and any subset $\Omega_{0} \subseteq \Omega$, it follows from $x_{i}^{k} \rightarrow \infty, \forall i \in J$, that

$$
\lim _{k \rightarrow \infty} y_{J}\left(x^{k}, \omega\right)=\lim _{k \rightarrow \infty}\left(M(\omega) x^{k}+q(\omega)\right)_{J}=0, \quad \text { a.e. } \quad \omega \in \Omega .
$$

Now consider the following quadratic programming problem:

$$
\min _{x \in R_{+}^{n}} g(x):=E\left[(M(\omega) x+q(\omega))_{J}^{T}(M(\omega) x+q(\omega))_{J}\right] .
$$

It is easy to see that $g(x) \geq 0$ for all $x \in R_{+}^{n}$. Hence, by the well-known Frank-Wolfe theorem [10], this problem attains its infimum in $R_{+}^{n}$. Furthermore, the infimum must be zero, since (2.13) implies $\lim _{k \rightarrow \infty} g\left(x^{k}\right)=0$. Therefore, there exists $\tilde{x} \in R_{+}^{n}$ such that

$$
(M(\omega) \tilde{x}+q(\omega))_{J}=0, \quad \text { a.e. } \quad \omega \in \Omega .
$$

Since $J \subseteq \bar{\alpha} \neq \emptyset$, this contradicts the assumption that for any $i \in \bar{\alpha}$, there is no $x \in R_{+}^{n}$ such that (2.10) holds.

Since $\gamma$ is arbitrary, we get the desired result.

Corollary 2.2 If the monotone $L C P(\bar{M}, \bar{q})$ has a nonempty and bounded solution set, then the ERM formulation (1.4) defined by the penalized FB function $\phi_{3}$ has a nonempty and bounded solution set.

It should be noticed that in Example 2.1, the solution set of the monotone $\operatorname{LCP}(\bar{M}, \bar{q})$ is unbounded, but $D_{3}(\gamma)$ is bounded for all $\gamma \geq 0$.

\subsection{Regularization}

To establish the solvability of the ERM formulation (1.4) for the monotone SLCP without assuming the boundedness of the solution set of the monotone $\operatorname{LCP}(\bar{M}, \bar{q})$, we consider a regularized version of (1.4). For $\epsilon>0$, let

$$
y(x, \omega, \epsilon):=(M(\omega)+\epsilon I) x+q(\omega)
$$

and

$$
\Phi(x, \omega, \epsilon):=\left(\begin{array}{c}
\phi\left(y_{1}(x, \omega, \epsilon), x_{1}\right) \\
\vdots \\
\phi\left(y_{n}(x, \omega, \epsilon), x_{n}\right)
\end{array}\right)
$$


The regularized problem for (1.4) is defined as

$$
\min _{x \in R_{+}^{n}} f(x, \epsilon):=E\left[\|\Phi(x, \omega, \epsilon)\|^{2}\right] .
$$

We will study the behavior of the sequence $\left\{x_{\epsilon_{k}}\right\}$ of solutions to (2.14) for an arbitrarily chosen positive sequence $\left\{\epsilon_{k}\right\}$ tending to zero. In the following, to simplify the notation, we will denote $\{\epsilon\}$ and $\left\{x_{\epsilon}\right\}$ for $\left\{\epsilon_{k}\right\}$ and $\left\{x_{\epsilon_{k}}\right\}$, respectively.

Theorem 2.3 Suppose $\bar{M}$ is positive semi-definite. Then for any $\epsilon>0$, the regularized problem (2.14) has a nonempty and bounded solution set $S_{E R M_{\epsilon}}$. Let $x_{\epsilon} \in S_{E R M_{\epsilon}}$ for each $\epsilon>0$. Then every accumulation point of the sequence $\left\{x_{\epsilon}\right\}$ is contained in the set $S_{E R M}$.

Proof: Note that $E[M(\omega)+\epsilon I]=\bar{M}+\epsilon I$ is positive definite. From Remark 2.1, the solution set $S_{E R M_{\epsilon}}$ of (2.14) defined by the "min" function is nonempty and bounded. Moreover, from Lemma 2.1, the solution set of the strongly monotone $\operatorname{LCP}(\bar{M}+\epsilon I, \bar{q})$ is nonempty and bounded; in fact, it is a singleton. Hence, by Theorem 2.2, the solution set $S_{E R M_{\epsilon}}$ of (2.14) defined by the penalized FB function is nonempty and bounded.

Let $\bar{x}$ be an accumulation point of $\left\{x_{\epsilon}\right\}$. For simplicity, we assume that $\left\{x_{\epsilon}\right\}$ itself converges to $\bar{x}$. Now we show

$$
\left|f\left(x_{\epsilon}, \epsilon\right)-f(\bar{x})\right| \rightarrow 0 \quad \text { as } \quad \epsilon \rightarrow 0 .
$$

From the continuity of $f$, we observe that

$$
\left|f\left(x_{\epsilon}\right)-f(\bar{x})\right| \rightarrow 0 \quad \text { as } \quad \epsilon \rightarrow 0 .
$$

Therefore, for (2.15), it is sufficient to show

$$
\left|f\left(x_{\epsilon}, \epsilon\right)-f\left(x_{\epsilon}\right)\right| \rightarrow 0 \quad \text { as } \quad \epsilon \rightarrow 0 .
$$

It is not difficult to verify that, for any $a, b \in R$ and $c \geq 0$,

$$
\left|\phi_{1}(b, a)-\phi_{1}(c, a)\right| \leq|b-c|, \quad \text { and } \quad\left|\phi_{3}(b, a)-\phi_{3}(c, a)\right| \leq\left(2+[a]_{+}\right)|b-c| .
$$

Hence, for any $x \in R^{n}$ and any $\omega \in \Omega$, we have

$$
\|\Phi(x, \omega, \epsilon)-\Phi(x, \omega)\| \leq(2+\|x\|)\|y(x, \omega, \epsilon)-y(x, \omega)\| \leq(2+\|x\|) \epsilon\|x\|
$$

and

$$
\begin{aligned}
\left|f\left(x_{\epsilon}, \epsilon\right)-f\left(x_{\epsilon}\right)\right| & =\left|E\left[\left\|\Phi\left(x_{\epsilon}, \omega, \epsilon\right)\right\|^{2}-\left\|\Phi\left(x_{\epsilon}, \omega\right)\right\|^{2}\right]\right| \\
& =\left|E\left[\left(\left\|\Phi\left(x_{\epsilon}, \omega, \epsilon\right)\right\|+\left\|\Phi\left(x_{\epsilon}, \omega\right)\right\|\right)\left(\left\|\Phi\left(x_{\epsilon}, \omega, \epsilon\right)\right\|-\left\|\Phi\left(x_{\epsilon}, \omega\right)\right\|\right)\right]\right| \\
& \leq E\left[\left(\left\|\Phi\left(x_{\epsilon}, \omega, \epsilon\right)\right\|+\left\|\Phi\left(x_{\epsilon}, \omega\right)\right\|\right)\left\|\Phi\left(x_{\epsilon}, \omega, \epsilon\right)-\Phi\left(x_{\epsilon}, \omega\right)\right\|\right] .
\end{aligned}
$$

Now choose $\delta>0$ arbitrarily and let $B:=\{x \mid\|x-\bar{x}\|<\delta\}$. Then, for any $x \in B$, we have $\|x\| \leq c_{0}:=\|\bar{x}\|+\delta$. Moreover, from Assumption I, there is a positive constant $c_{1}$ such that for any $x \in B$,

$$
E[\|\Phi(x, \omega)\|] \leq c_{1} .
$$


Since $x_{\epsilon} \rightarrow \bar{x}$, there is a small $\epsilon_{0}>0$ such that $x_{\epsilon} \in B$ for all $\epsilon \in\left(0, \epsilon_{0}\right)$. Therefore, from (2.17) and (2.18), we have

$$
\begin{aligned}
\left|f\left(x_{\epsilon}, \epsilon\right)-f\left(x_{\epsilon}\right)\right| & \leq\left(2 E\left[\left\|\Phi\left(x_{\epsilon}, \omega\right)\right\|\right]+(2+\|x\|) \epsilon\|x\|\right)(2+\|x\|) \epsilon\|x\| \\
& \leq\left(2 c_{1}+\left(2+c_{0}\right) c_{0} \epsilon\right)\left(2+c_{0}\right) c_{0} \epsilon .
\end{aligned}
$$

Letting $\epsilon \rightarrow 0$, we obtain (2.16) and hence (2.15). Furthermore, for every $x \in R_{+}^{n}$, from (2.15) and the inequality

$$
f(\bar{x})=\lim _{\epsilon \downarrow 0} f\left(x_{\epsilon}, \epsilon\right) \leq \lim _{\epsilon \downarrow 0} f(x, \epsilon)=f(x),
$$

we find that $\bar{x} \in S_{E R M}$.

To compare (1.4) with the regularized problem (2.14), we consider the following example.

Example 2.4 [3] Let $M(\cdot)$ and $q(\cdot)$ be defined as in Example 2.1. We have

$$
f_{1}(x)=\frac{1}{2} \begin{cases}2, & 1 \leq x \\ 1+x^{2}, & 0 \leq x \leq 1\end{cases}
$$

and for $\epsilon \in(0,1)$,

$$
f_{1}(x, \epsilon)=\frac{1}{2} \begin{cases}(\epsilon x-1)^{2}+(\epsilon x+1)^{2}, & \frac{1}{1-\epsilon}<x \\ (\epsilon x-1)^{2}+x^{2}, & 0 \leq x \leq \frac{1}{1-\epsilon} .\end{cases}
$$

Obviously $x^{*}=0$ is the unique global minimizer of (1.4), and any $x \in\{x \mid x>1\}$ is a local minimizer of (1.4). For any $\epsilon \in(0,1)$, the regularized problem has a unique local (global) minimizer $x_{\epsilon}=\frac{\epsilon}{1+\epsilon^{2}}$. Letting $\epsilon \rightarrow 0$, we have $x_{\epsilon} \rightarrow x^{*}=0$. This example shows that in general $f(x)$ is not a convex function and may have many local minimizers. Hence the regularization method may be helpful in finding a global solution.

We should clarify the meaning of the conclusion of Theorem 2.3. The result applies regardless of whether the sequence $\left\{x_{\epsilon}\right\}$ has an accumulation point or not. In the case $\left\{x_{\epsilon}\right\}$ is bounded, $\left\{x_{\epsilon}\right\}$ has an accumulation point. In the opposite case, we do not know if it has an accumulation point. Now we give sufficient conditions for $\left\{x_{\epsilon}\right\}$ to be bounded.

Theorem 2.4 Under the assumptions of Theorem 2.2, the solution sequence $\left\{x_{\epsilon}\right\}$ of the regularized problem (2.14) defined by the penalized FB function is bounded.

Proof: Suppose on the contrary that $\left\{x_{\epsilon}\right\} \subset R_{+}^{n}$ is unbounded. First we observe that the sequence $\left\{f_{3}\left(x_{\epsilon}, \epsilon\right)\right\}$ is bounded since for any $\epsilon \geq 0$,

$$
0 \leq f_{3}\left(x_{\epsilon}, \epsilon\right) \leq f_{3}(0, \epsilon)=f_{3}(0)
$$

Then, in a similar manner to the proof of Lemma 2.3, we can show that for any index $i \in \mathcal{N}$ and any subset $\Omega_{0} \subseteq \Omega$, the sequence $\left\{E\left[y_{i}\left(x_{\epsilon}, \omega, \epsilon\right) 1_{\left\{\omega \in \Omega_{0}\right\}}\right]\right\}$ is bounded below, and 
the sequence $\left\{\left(x_{\epsilon}\right)_{i} E\left[y_{i}\left(x_{\epsilon}, \omega, \epsilon\right) 1_{\left\{\omega \in \Omega_{0}\right\}}\right]\right\}$ is bounded above. By choosing a subsequence of $\left\{x_{\epsilon}\right\}$ if necessary, we define the index set $J=\left\{i \mid\left(x_{\epsilon}\right)_{i} \rightarrow \infty\right\}$.

Denote $\bar{y}_{\epsilon}=E\left[y\left(x_{\epsilon}, \omega, \epsilon\right)\right]=\bar{M} x_{\epsilon}+\epsilon x_{\epsilon}+\bar{q}$. Let $\hat{x} \in \mathcal{F}$ be arbitrarily chosen, and put $\hat{y}=\bar{M} \hat{x}+\bar{q}$. Then there exists a constant $\gamma>0$, such that for any $\epsilon>0$ small enough,

$$
\begin{aligned}
\gamma \geq\left(x_{\epsilon}-\hat{x}\right)^{T}\left(\bar{y}_{\epsilon}-\hat{y}\right) & =\left(x_{\epsilon}-\hat{x}\right)^{T} \bar{M}\left(x_{\epsilon}-\hat{x}\right)+\epsilon x_{\epsilon}^{T}\left(x_{\epsilon}-\hat{x}\right) \\
& \geq \epsilon x_{\epsilon}^{T}\left(x_{\epsilon}-\hat{x}\right) \geq 0,
\end{aligned}
$$

where the first inequality uses the fact that $\left\{x_{\epsilon} \bar{y}_{\epsilon}\right\}$ is bounded above, $\left\{\bar{y}_{\epsilon}\right\}$ is bounded below and $\hat{x} \geq 0, \hat{y} \geq 0, x_{\epsilon} \geq 0$, while the next two inequalities use the positive semidefiniteness of $\bar{M}$ and $J \neq \emptyset$, respectively. Thus, from (2.19) and $\left\|x_{\epsilon}\right\| \rightarrow \infty$, we have

$$
0 \leq \lim _{\epsilon \downarrow 0} \epsilon\left\|x_{\epsilon}\right\| \leq \lim _{\epsilon \downarrow 0} \frac{\gamma+\epsilon x_{\epsilon}^{T} \hat{x}}{\left\|x_{\epsilon}\right\|}=0,
$$

which together with (2.19) yields that $\left\{\epsilon x_{\epsilon}^{T} x_{\epsilon}\right\}$ is bounded. By Cauchy-Schwartz inequality, we obtain

$$
E\left[\left\|\Phi_{3}\left(x_{\epsilon}, \omega, \epsilon\right)\right\|\right] \leq \sqrt{E\left[\left\|\Phi_{3}\left(x_{\epsilon}, \omega, \epsilon\right)\right\|^{2}\right]}=\sqrt{f_{3}\left(x_{\epsilon}, \epsilon\right)} \leq \sqrt{f_{3}(0)} .
$$

Thus, the sequence $\left\{E\left[\left\|\Phi_{3}\left(x_{\epsilon}, \omega, \epsilon\right)\right\|\right]\right\}$ is bounded. According to (2.17) and (2.18) in the proof of Theorem 2.3, we have

$$
\left|f_{3}\left(x_{\epsilon}, \epsilon\right)-f_{3}\left(x_{\epsilon}\right)\right| \leq\left(2 E\left[\left\|\Phi_{3}\left(x_{\epsilon}, \omega, \epsilon\right)\right\|\right]+\left(2+\left\|x_{\epsilon}\right\|\right) \epsilon\left\|x_{\epsilon}\right\|\right)\left(2+\left\|x_{\epsilon}\right\|\right) \epsilon\left\|x_{\epsilon}\right\|,
$$

which implies that there exists a constant $\gamma_{2}>0$ such that $f_{3}\left(x_{\epsilon}\right) \leq \gamma_{2}$ for any $\epsilon>0$. Then, from Theorem 2.2, we conclude that the sequence $\left\{x_{\epsilon}\right\}$ is bounded.

Remark 2.2 In general, the function $f(x)$ and its regularization $f(x, \epsilon)$ is not convex. Regarding standard results for $L C P(\bar{M}, \bar{q})$, several interesting questions remain to be studied: Does $S_{E R M_{\epsilon}}$ have only one element? Is the whole sequence $\left\{x_{\epsilon}\right\}$ convergent if there is a convergent subsequence?

\section{Robust solution}

The EV formulation and the ERM formulation take into account all random events and give decisions under uncertainty. In general, the decisions may not be the best or may be even infeasible for each individual event. However, in many cases, we have to take risk to make a priori decision based on limited information of unknown random events. Naturally, one wants to know how good or how bad the decision given by a deterministic formulation can be. In this section, we study the robustness of solutions of the ERM formulation (1.4) for the monotone SLCP.

Let $\bar{\Phi}$ be defined by (2.4). For any $x$, by taking expectation in

$$
\|\Phi(x, \omega)\|^{2}=\|\bar{\Phi}(x)\|^{2}+2 \bar{\Phi}(x)^{T}(\Phi(x, \omega)-\bar{\Phi}(x))+\|\Phi(x, \omega)-\bar{\Phi}(x)\|^{2},
$$


we find

$$
f(x)=E\left[\|\Phi(x, \omega)\|^{2}\right]=\|\bar{\Phi}(x)\|^{2}+E\left[\|\Phi(x, \omega)-\bar{\Phi}(x)\|^{2}\right] .
$$

Note that the second term

$$
\begin{aligned}
E\left[\|\Phi(x, \omega)-\bar{\Phi}(x)\|^{2}\right] & =E\left[\operatorname{tr}(\Phi(x, \omega)-\bar{\Phi}(x))(\Phi(x, \omega)-\bar{\Phi}(x))^{T}\right] \\
& =\operatorname{tr} E\left[(\Phi(x, \omega)-\bar{\Phi}(x))(\Phi(x, \omega)-\bar{\Phi}(x))^{T}\right]
\end{aligned}
$$

is the trace of the covariance matrix of the random function $\Phi(x, \omega)$.

Since $\Phi(x, \omega)=0$ if and only if $x$ solves $\operatorname{LCP}(M(\omega), q(\omega))$, and the ERM formulation (1.4) is equivalent to

$$
\min _{x \in R_{+}^{n}}\|\bar{\Phi}(x)\|^{2}+E\left[\|\Phi(x, \omega)-\bar{\Phi}(x)\|^{2}\right],
$$

an optimal solution of the ERM formulation (1.4) yields a high mean performance of the SLCP and has a minimum sensitivity with respect to random parameter variations in SLCP. Therefore, the ERM formulation (1.4) can be regarded as a robust formulation for SLCP.

Now, we investigate the relation between a solution of the ERM formulation and a solution of $\operatorname{LCP}(M(\omega), q(\omega))$ for $\omega \in \Omega$. First, we give a new error bound for monotone LCP which uses the sum of the "min" function $\phi_{1}(a, b)$ and the penalized FB function $\phi_{3}(a, b)$. The idea comes from the error bound given by Mangasarian and Ren [17]. Let $\operatorname{SOL}(A, p)$ denote the solution set of $\operatorname{LCP}(A, p)$, and define the distance from a point $x$ to the set $\operatorname{SOL}(A, p)$ by $\operatorname{dist}(x, \operatorname{SOL}(A, p)):=\|x-\bar{x}(x)\|$, where $\bar{x}(x)$ is a closest solution of $\operatorname{LCP}(A, p)$ to $x$ under the norm $\|\cdot\|$. Let

$$
\Psi_{1}(x):=\|\min (x, A x+p)\|
$$

and

$$
s(x):=\left\|\left[-A x-p,-x, x^{T}(A x+p)\right]_{+}\right\| .
$$

Lemma 3.1 [17] Suppose that $A$ is positive semi-definite and $\operatorname{SOL}(A, p) \neq \emptyset$. Then there is a constant $c>0$ such that

$$
\operatorname{dist}(x, \operatorname{SOL}(A, p)) \leq c\left(\Psi_{1}(x)+s(x)\right), \quad x \in R^{n} .
$$

Lemma 3.2 Let $\psi(a, b)=[-b,-a, a b]_{+}$. Then we have $\|\psi(a, b)\| \leq\left|\phi_{3}(a, b)\right|$ for any $a \geq 0$ and $b \in R$.

Proof: Let $a \geq 0$. If $b \geq 0$, then from $a+b \geq \sqrt{a^{2}+b^{2}}$, we have

$$
\|\psi(a, b)\|=a b \leq a+b-\sqrt{a^{2}+b^{2}}+a b=\left|\phi_{3}(a, b)\right| .
$$

If $b<0$, then from $a \leq \sqrt{a^{2}+b^{2}}$, we have $a+b-\sqrt{a^{2}+b^{2}} \leq b<0$, and

$$
\|\psi(a, b)\|=|-b| \leq\left|a+b-\sqrt{a^{2}+b^{2}}\right|=\left|\phi_{3}(a, b)\right| .
$$


From Lemma 3.2, it is easy to see that for any $x \geq 0$,

$$
s(x) \leq \Psi_{3}(x):=\left\|\left(\phi_{3}\left(x_{1},(A x+p)_{1}\right), \ldots, \phi_{3}\left(x_{n},(A x+p)_{n}\right)\right)\right\| .
$$

Moreover, from (2.3), there is a constant $\kappa>0$ such that

$$
\Psi_{1}(x) \leq \kappa \Psi_{3}(x), \quad x \in R^{n} .
$$

Using these inequalities with Lemma 3.1, we obtain the following new global error bounds for the monotone $\operatorname{LCP}(A, p)$.

Theorem 3.1 Let the monotone $L C P(A, p)$ have a nonempty solution set $\operatorname{SOL}(A, p)$. Then both $\Psi_{1}+\Psi_{3}$ and $\Psi_{3}$ provide global error bounds for the monotone LCP on $R_{+}^{n}$, that is, there are positive constants $\alpha_{1}$ and $\alpha_{2}$ such that

$$
\operatorname{dist}(x, \operatorname{SOL}(A, p)) \leq \alpha_{1}\left(\Psi_{1}(x)+\Psi_{3}(x)\right) \leq \alpha_{2} \Psi_{3}(x), \quad x \in R_{+}^{n} .
$$

To give error bounds for SLCP, we assume that $M(\omega)$ is a positive semi-definite matrix and $\operatorname{LCP}(M(\omega), q(\omega))$ has a nonempty solution set for every $\omega \in \Omega$. This assumption holds in many applications. For instance, consider the stochastic quadratic program

$$
\begin{aligned}
\min & \frac{1}{2} z^{T} Q z+c^{T} z \\
\text { s.t } & A(\omega) z \geq b(\omega), z \geq 0,
\end{aligned}
$$

where $Q$ is a positive definite matrix. The KKT conditions for this quadratic program yield the SLCP involving the random matrix

$$
M(\omega)=\left(\begin{array}{cc}
Q & -A(\omega)^{T} \\
A(\omega) & 0
\end{array}\right) .
$$

Clearly this is a positive semi-definite matrix for each $\omega$.

Theorem 3.2 Assume that $\Omega=\left\{\omega^{1}, \omega^{2} \ldots, \omega^{N}\right\} \subset R^{m}$, and for every $\omega \in \Omega, M(\omega)$ is a positive semi-definite matrix and $\operatorname{LCP}(M(\omega), q(\omega))$ has a nonempty solution set. Then there are positive constants $\beta_{1}$ and $\beta_{2}$ such that

$$
E[\operatorname{dist}(x, \operatorname{SOL}(M(\omega), q(\omega)))] \leq \beta_{1}\left(\sqrt{f_{1}(x)}+\sqrt{f_{3}(x)}\right) \leq \beta_{2} \sqrt{f_{3}(x)}, \quad x \in R_{+}^{n} .
$$

Theorem 3.2 particularly shows that for $x^{*} \in S_{E R M}$,

$$
E\left[\operatorname{dist}\left(x^{*}, \operatorname{SOL}(M(\omega), q(\omega))\right)\right] \leq \beta_{2} \sqrt{f_{3}\left(x^{*}\right)}=\beta_{2} \min _{x \in R_{+}^{n}} \sqrt{f_{3}(x)} .
$$

Unlike an error bound for the deterministic LCP, the left-hand side of (3.2) is in general positive at a solution of the ERM formulation (1.4). Nevertheless, the inequality (3.2) suggests that the expected distance to the solution set $\operatorname{SOL}(M(\omega), q(\omega))$ for $\omega \in \Omega$ is also likely to be small at $x^{*} \in S_{E R M}$. In other words, we may expect that a solution of the ERM formulation (1.4) has a minimum sensitivity with respect to random parameter variations in SLCP. In this sense, solutions of (1.4) can be regarded as robust solutions for SLCP. 


\section{Numerical experiments}

We have conducted some numerical experiments to investigate the properties of solutions of the ERM formulation (1.4) for monotone SLCP. In particular, we have made comparison of the ERM formulation with the EV formulation (1.5) in terms of the measures of optimality and feasibility as well as that of reliability, which are defined through a quadratic programming formulation of SLCP.

We start with some preliminary materials about calculations of gradients and Hessian matrices of functions $f_{1}$ and $f_{3}$ in the ERM formulation (1.4).

\subsection{Gradient and Hessian}

If the strict complementarity condition holds with probability one at $x$, then $f_{1}$ is twice continuously differentiable at $x$. In this case, the gradient $g_{1}(x)$ of $f_{1}$ is given by

$$
g_{1}(x)=E\left[M(\omega)^{T}(I-D(x, \omega))(M(\omega) x+q(\omega))+(I+D(x, \omega)) x\right]
$$

and the Hessian matrix $G_{1}(x)$ of $f_{1}$ is given by

$$
G_{1}(x)=E\left[M(\omega)^{T}(I-D(x, \omega)) M(\omega)+I+D(x, \omega)\right],
$$

where $D(x, \omega)=\operatorname{diag}(\operatorname{sign}(M(\omega) x+q(\omega)-x))$.

The function $f_{3}$ defined by the penalized FB function (2.1) with $\lambda \in(0,1)$ is continuously differentiable at any point $x \in R^{n}$, and twice continuously differentiable at point $x$ where $\mathcal{P}\left\{\omega \mid x_{i}=y_{i}(x, \omega)=0, i=1, \ldots, n\right\}=0$. The gradient $g_{3}(x)$ of $f_{3}$ is given by

$$
g_{3}(x)=E\left[\nabla\left\|\Phi_{3}(x, \omega)\right\|^{2}\right]=2 E\left[V(x, \omega)^{T} \Phi_{3}(x, \omega)\right]
$$

where $V(x, \omega) \in R^{n \times n}$ can be computed by Algorithm 1 in [2].

If $f_{3}$ is twice continuously differentiable at $x$, then the Hessian matrix $G_{3}(x)$ is given by

$$
G_{3}(x)=E\left[\nabla^{2}\left\|\Phi_{3}(x, \omega)\right\|^{2}\right]=2 E\left[V(x, \omega)^{T} V(x, \omega)+\sum_{i=1}^{n} U_{i}(x, \omega)\left(\Phi_{3}(x, \omega)\right)_{i}\right],
$$

where $\sum_{i=1}^{n} U_{i}(x, \omega)\left(\Phi_{3}(x, \omega)\right)_{i}$ can be computed as follows:

For each $\omega \in \Omega$, define vectors $\xi, \eta, c_{x}, c_{x y}, c_{y} \in R^{n}$ with components

$$
\begin{aligned}
\xi_{i} & =\left(x_{i}^{2}+y_{i}(x, \omega)^{2}\right)^{-\frac{3}{2}}, \quad \eta_{i}=\operatorname{sign}\left(\left[x_{i}\right]_{+}\left[y_{i}(x, \omega)\right]_{+}\right), \\
\left(c_{x}\right)_{i} & =\xi_{i} x_{i}^{2}, \quad\left(c_{x y}\right)_{i}=\xi_{i} x_{i} y_{i}(x, \omega), \quad\left(c_{y}\right)_{i}=\xi_{i} y_{i}(x, \omega)^{2} .
\end{aligned}
$$

Denote matrices $D_{\phi}=\operatorname{diag}\left(\Phi_{3}(x, \omega)\right), D_{\lambda}=\operatorname{diag}\left(\lambda c_{x y}+(1-\lambda) \eta\right), D_{y}=\operatorname{diag}\left(c_{y}\right), \tilde{W}=$ $D_{\phi} D_{\lambda} M(\omega)$, and $W_{i}=M_{i}(\omega)^{T} M_{i}(\omega)$ for $i=1, \ldots, n$, then

$$
\sum_{i=1}^{n} U_{i}(x, \omega)\left(\Phi_{3}(x, \omega)\right)_{i}=\tilde{W}+\tilde{W}^{T}-\lambda \sum_{i=1}^{n}\left(D_{\phi} c_{x}\right)_{i} W_{i}-\lambda D_{y} D_{\phi} .
$$




\subsection{Measure of optimality and feasibility}

Using reformulations of LCP and stochastic programming techniques, we may consider various deterministic formulations of SLCP. For instance, in a recent paper [15], another approach for stochastic nonlinear complementarity problems has been proposed as an application of stochastic mathematical programs with equilibrium constraints. Different deterministic formulations of SLCP have different optimal solutions. To help decision makers to select a proper solution, we introduce some measure of optimality and feasibility for a given point $x \in R_{+}^{n}$.

As stated in the introduction, the function value $f(x)$ can be regarded as an expected total cost. Let $x^{*}$ be a solution of (1.4) with $\Omega=\left\{\omega^{1}, \omega^{2}, \ldots, \omega^{N}\right\}$. By the definition of ERM formulation, there is no $x \in R_{+}^{n}$ such that

$$
\mathcal{P}\left\{\omega \mid\|\Phi(x, \omega)\|<\left\|\Phi\left(x^{*}, \omega\right)\right\|\right\}=1 .
$$

Hence $x^{*}$ is a weak Pareto optimal solution of the SLCP in the sense of multi-objective optimization

$$
\min _{x \in R_{+}^{n}}\left(\begin{array}{c}
\left\|\Phi\left(x, \omega^{1}\right)\right\| \\
\vdots \\
\left\|\Phi\left(x, \omega^{N}\right)\right\|
\end{array}\right) .
$$

Now we define some measure of optimality and feasibility for a given point $x$, without using an NCP function. For a fixed $\omega, \operatorname{LCP}(M(\omega), q(\omega))$ is equivalent to the quadratic program

$$
\begin{aligned}
\min & y(x, \omega)^{T} x \\
\text { s.t } & y(x, \omega):=M(\omega) x+q(\omega) \geq 0, x \geq 0
\end{aligned}
$$

in the sense that (4.1) has an optimal solution with zero objective value if and only if $\operatorname{LCP}(M(\omega), q(\omega))$ has a solution. We adopt some ideas of loss functions from the literature of stochastic programming $[1,12,18]$ to problem (4.1). For $x \in R_{+}^{n}$, let

$$
\gamma(x, \omega):=\|\min (0, y(x, \omega))\|+x^{T}[y(x, \omega)]_{+} .
$$

It is easy to verify that $x_{\omega}$ is a solution of (4.1) if and only if $\gamma\left(x_{\omega}, \omega\right)=0$ and $x_{\omega} \geq 0$, provided $\operatorname{LCP}(M(\omega), q(\omega))$ has a solution. In (4.2), the first term evaluates violation of the nonnegativity condition and the second term evaluates the loss in the objective function of (4.1). For a fixed $x \in R_{+}^{n}$, the expected total loss is defined by $E[\gamma(x, \omega)]$. For two points $x^{*}, \bar{x} \in R_{+}^{n}$, we define the measure of dominance of $x^{*}$ over $\bar{x}$ by

$$
\pi\left(x^{*}, \bar{x}\right):=\mathcal{P}\left\{\omega \mid \gamma\left(x^{*}, \omega\right)<\gamma(\bar{x}, \omega)\right\} .
$$

If $\pi\left(x^{*}, \bar{x}\right)>0.5$, then $x^{*}$ has more chance to dominate $\bar{x}$, and so $x^{*}$ may be regarded as a better point than $\bar{x}$ in the multi-objective optimization problem

$$
\min _{x \in R_{+}^{n}}\left(\begin{array}{c}
\gamma\left(x, \omega^{1}\right) \\
\vdots \\
\gamma\left(x, \omega^{N}\right)
\end{array}\right) .
$$


In many engineering and economic applications of SLCP, the inequality $y(x, \omega) \geq 0$ describes the safety of the system, and the guarantee of safety is critically important. Under those circumstances, we may judge that a failure occurs if and only if there is an index $i$ such that $y_{i}(x, \omega)<0$. Let

$$
y^{\min }(x, \omega):=\min _{1 \leq i \leq n} y_{i}(x, \omega) .
$$

The reliability of $x$ with a tolerance $\epsilon>0$ is then defined by

$$
\operatorname{rel}_{\epsilon}(x):=\mathcal{P}\left\{\omega \mid y^{\min }(x, \omega) \geq-\epsilon\right\}
$$

\subsection{Test problems}

We give a procedure to generate a test problem of the ERM formulation for discretized monotone SLCP,

$$
\min _{x \in R_{+}^{n}} f(x):=\frac{1}{N} \sum_{j=1}^{N} \sum_{i=1}^{n} \phi\left(x_{i},\left(M^{j} x+q^{j}\right)_{i}\right)^{2},
$$

where $M^{j}=M\left(\omega^{j}\right)$ and $q^{j}=q\left(\omega^{j}\right)$ for $j=1, \ldots, N$ and $\Omega=\left\{\omega^{1}, \omega^{2}, \ldots, \omega^{N}\right\}$.

Let $\hat{x}$ be a nominal point chosen in $R_{+}^{n}$, which is used as a seed of constructing a set of test problems and becomes a solution of the ERM formulation (1.4) in some special cases (see below for the detail). Moreover, the user is required to specify the following parameters:

- $n$ : the number of variables

- $N$ : the number of random matrices and vectors

- $\mu^{2}(\mu \geq 1)$ : the condition number of the expectation matrix $\bar{M}$

- $n_{x}$ : the number of elements in the index set $\mathcal{J}=\left\{i \mid \hat{x}_{i}>0\right\}$

- $(0, \tau)$ : the range of $\hat{x}_{i}$ for $i \in \mathcal{J}$

- \# $I_{j}$ : the number of elements in the index set $\mathcal{I}_{j}=\left\{i \mid \hat{x}_{i}=0,\left(M^{j} \hat{x}+q^{j}\right)_{i}>0\right\}$ for each $j$

- \#K$K_{j}$ : the number of elements in the index set $\mathcal{K}_{j}=\left\{i \mid \hat{x}_{i}=0,\left(M^{j} \hat{x}+q^{j}\right)_{i}=0\right\}$ for each $j$

- $(0, \nu)$ : the range of $\left(M^{j} \hat{x}+q^{j}\right)_{i}$ for $i \in \mathcal{I}_{j}$ and each $j$

- $[0, \beta)$ : the range of $\left(M^{j} \hat{x}+q^{j}\right)_{i}$ for $i \in \mathcal{J}$

- $(-\sigma, \sigma)$ : the range of elements of matrix $\bar{M}-M^{j}$ for each $j$

\section{Procedure for generating a test problem of monotone SLCP}

1. Randomly generate a vector $\hat{x} \in R_{+}^{n}$ that has $n_{x}$ positive elements in $(0, \tau)$. 
2. Generate a diagonal matrix $D$ whose diagonal elements are determined as

$$
D_{i i}= \begin{cases}1 / \mu & i=1 \\ \mu^{\lambda_{i}} & i=2, \ldots, n-1 \\ \mu & i=n,\end{cases}
$$

where $\lambda_{i}, i=2, \ldots, n-1$ are uniform variate in the interval $(-1,1)$.

3. Generate a random orthogonal matrix $U \in R^{n \times n}$ by using the singular value decomposition of a random matrix, and let $\bar{M}=U D U^{T}$.

4. Generate $N$ random matrices $B^{j} \in R^{n \times n}, j=1,2, \ldots, N$ whose elements are in the interval $(0,1)$. Set

$$
M^{j}=\bar{M}+\sigma\left(B^{j}-B^{N-j+1}\right), \quad j=1,2, \ldots, N .
$$

5. For each $j=1,2, \ldots, N$, set

$$
q_{i}^{j}= \begin{cases}\left(-M^{j} \hat{x}\right)_{i} & i \in \mathcal{K}_{j} \\ \left(-M^{j} \hat{x}+\beta z^{j}\right)_{i} & i \in \mathcal{J} \\ \left(-M^{j} \hat{x}+\nu z^{j}\right)_{i} & i \in \mathcal{I}_{j},\end{cases}
$$

where $z^{j} \in R^{n}$ is a random vector whose elements are in the interval $(0,1)$.

\section{Some aspects of the test problem}

- The expectation matrix $\bar{M}=U D U^{T}$ is symmetric positive definite. Its condition number is $\mu^{2}$ and its eigenvalues are distributed on the interval $[1 / \mu, \mu]$.

- If $\sigma=0$, then all $M^{j}$ are equal to $\bar{M}=U D U^{T}$, which is positive definite. For $\sigma>0, M^{j}$ may not be a positive semi-definite matrix, but $\left|\left(\bar{M}-M^{j}\right)_{i l}\right|=\sigma \mid\left(B^{j}-\right.$ $\left.B^{N-j+1}\right)_{i l} \mid \leq \sigma$ for all $i, l=1, \ldots, n$.

- If $\# K_{j}=0$ for all $j=1, \ldots, N$, then $f_{1}$ is continuously differentiable at $\hat{x}$.

- If $\beta=0$, then $\hat{x}$ is a solution of $\operatorname{LCP}\left(M^{j}, q^{j}\right)$ for all $j=1,2, \ldots, N$. In this case, $\hat{x}$ becomes a global solution of (4.4) with $f(\hat{x})=\min _{x \in R_{+}^{n}} f(x)=0$.

- $n-n_{x}$ is the number of active constraints at $\hat{x}$.

- If $\beta>0$, then we have in general $f(\hat{x})>0$. In this case, $\hat{x}$ is not necessarily a solution of (4.4). However, by Remark 2.1 and Theorem 2.2, the positive definiteness of $\bar{M}$ guarantees that the solution set of (4.4) is nonempty and bounded. 


\subsection{Numerical results}

We used the program of Lemke's method [8] to get a solution $\bar{x}$ of the EV formulation (1.5). To solve the ERM formulation (4.4), we used a semi-smooth Newton method with descent direction line search [6]. In particular, we first applied a global descent line search with the gradient $\nabla f(x)$ to make the function value sufficiently decrease and get a rough approximate solution. Next, we used a local semi-smooth Newton method with the rough approximate solution as an initial point to get an approximate local optimal solution. As the ERM problem defined by the "min" function is nonsmooth, in a few occasions, the method failed to decrease the function value. When it happened, we restarted the method. All computations were carried out by using MATLAB on a PC.

We first tested our program on hundreds of random problems with $\beta=0$ generated by the procedure in the last subsection with different parameters $\left(n, N, \mu, n_{x}, \nu, \sigma\right)$ and starting points $x^{0}=\ell e$ where $\ell=0,10, \ldots, 50$ and $e$ is the $n$-dimensional vector of ones. Since $\beta=0$, the solution $x^{*}$ of (4.4) coincides with the nominal point $\hat{x}$. We have observed that the average function values and relative errors at computed solutions $\tilde{x}$ of (4.4) satisfy

$$
f(\tilde{x}) \leq 10^{-26}, \quad \frac{\left\|x^{*}-\tilde{x}\right\|}{\left\|x^{*}\right\|} \leq 10^{-17},
$$

which indicates that our method works successfully in finding a global solution of (4.4).

Next, for each fixed $\left(n, n_{x}, \beta, \sigma\right)$ with $\beta>0$, we used the procedure described in the previous subsection to generate 100 test problems with the following parameters:

$$
\tau=20, \mu=10, \nu=15, N=10^{3} .
$$

The number of elements in the index set $\mathcal{K}_{j}$ was determined by using a random number as $\# K_{j}=$ floor $\left(\left(n-n_{x}\right) \operatorname{rand}(1, N)\right)$. The numbers shown in Tables 4.1 and 4.2 are average values for the 100 problems.

In these tables, $x^{i}$ is the computed solution, where the index $i=1$ stands for the "min" function, and $i=3$ stands for the penalized FB function.

For any $x, \tilde{x} \in R_{+}^{n}$, we define $\Gamma(x):=E[\gamma(x, \omega)], \pi(x, \tilde{x})$ and $\operatorname{rel}_{\epsilon}(x)$ as follows:

$$
\begin{array}{ll}
\Gamma(x):=\frac{1}{N} \sum_{j=1}^{n} \gamma^{j}(x), & \gamma^{j}(x)=\left\|\min \left(0, y^{j}(x)\right)\right\|+x^{T}\left[y^{j}(x)\right]_{+}, \\
\pi(x, \tilde{x}):=\sum_{j=1}^{N} p_{j}, & p_{j}= \begin{cases}\frac{1}{N} & \text { if } \gamma^{j}(x)<\gamma^{j}(\tilde{x}) \\
0 & \text { otherwise }\end{cases} \\
\operatorname{rel}_{\epsilon}(x):=\sum_{j=1}^{N} p_{j}, & p_{j}= \begin{cases}\frac{1}{N} & \text { if } \min _{1 \leq i \leq n} y_{i}^{j}(x) \geq-\epsilon \\
0 & \text { otherwise. }\end{cases}
\end{array}
$$

where $y^{j}(x)=M^{j} x+q^{j}, j=1, \ldots, N$. 
Table 4.1 Function values and $r e l_{\epsilon}$ with $\epsilon=0$ (left) and $\epsilon=1$ (right).

\begin{tabular}{|c|c|c|c|c|c|c|c|}
\hline$\left(n, n_{x}, \beta, \sigma\right)$ & $f_{1}\left(x^{1}\right)$ & $f_{1}(\bar{x})$ & $f_{3}\left(x^{3}\right)$ & $f_{3}(\bar{x})$ & $\operatorname{rel}_{\epsilon}(\bar{x})$ & $\operatorname{rel}_{\epsilon}\left(x^{1}\right)$ & $\operatorname{rel}_{\epsilon}\left(x^{3}\right)$ \\
\hline $20,10,10,20$ & 254.87 & $2.13 \mathrm{e} 6$ & 447.82 & $1.05 \mathrm{e} 7$ & 0,0 & $0.55,0.91$ & $0.55,0.92$ \\
\hline $20,10,10,10$ & 241.89 & $4.47 \mathrm{e} 5$ & 448.99 & $2.13 \mathrm{e} 6$ & 0,0 & $0.55,0.91$ & $0.55,0.92$ \\
\hline $20,10,5,10$ & 69.41 & $2.62 \mathrm{e} 5$ & 131.64 & $1.34 \mathrm{e} 6$ & 0,0 & $0.54,0.96$ & $0.52,0.93$ \\
\hline $20,10,5,0$ & 18.89 & 75.78 & 32.69 & 154.36 & $0.31,0.37$ & $0.27,0.60$ & $0.21,0.51$ \\
\hline $40,20,10,20$ & 527.19 & $6.83 \mathrm{e} 6$ & 998.75 & $3.01 \mathrm{e} 7$ & 0,0 & $0.52,0.97$ & $0.52,0.97$ \\
\hline $40,20,10,10$ & 510.84 & $1.90 \mathrm{e} 6$ & 999.39 & $8.52 \mathrm{e} 6$ & 0,0 & $0.49,0.85$ & $0.49,0.84$ \\
\hline $40,20,5,10$ & 144.06 & $1.14 \mathrm{e} 6$ & 270.48 & $4.65 \mathrm{e} 6$ & 0,0 & $0.52,0.99$ & $0.50,0.98$ \\
\hline $40,20,5,0$ & 44.11 & 171.25 & 79.86 & 79.86 & $0.07,0.58$ & $0.05,0.58$ & $0.05,0.58$ \\
\hline $60,30,10,20$ & 812.27 & $1.29 \mathrm{e} 7$ & 1465.60 & $5.19 \mathrm{e} 7$ & 0,0 & $0.49,0.95$ & $0.49,0.95$ \\
\hline $60,30,10,10$ & 819.21 & $9.23 \mathrm{e} 6$ & 1442.70 & $4.39 \mathrm{e} 7$ & 0,0 & $0.45,0.79$ & $0.46,0.81$ \\
\hline $60,30,5,10$ & 215.60 & $1.77 \mathrm{e} 6$ & 418.87 & $7.05 \mathrm{e} 6$ & 0,0 & $0.38,0.99$ & $0.36,0.98$ \\
\hline $60,30,5,0$ & 58.29 & 281.16 & 100.56 & 576.09 & $0.51,0.58$ & $0.37,0.56$ & $0.28,0.48$ \\
\hline
\end{tabular}

Table 4.2 Relative dominance of solutions based on the stochastic QP formulation

\begin{tabular}{|c|c|c|c|c|c|c|c|}
\hline$\left(n, n_{x}, \beta, \sigma\right)$ & $\pi\left(x^{1}, \bar{x}\right)$ & $\pi\left(x^{3}, \bar{x}\right)$ & $\pi\left(x^{1}, x^{3}\right)$ & $\pi\left(x^{3}, x^{1}\right)$ & $\Gamma(\bar{x})$ & $\Gamma\left(x^{1}\right)$ & $\Gamma\left(x^{3}\right)$ \\
\hline $20,10,10,20$ & 1 & 1 & 0.49 & 0.51 & $3.67 \mathrm{e} 4$ & 518.13 & 517.91 \\
\hline $20,10,10,10$ & 1 & 1 & 0.49 & 0.51 & $1.56 \mathrm{e} 4$ & 491.21 & 490.64 \\
\hline $20,10,5,10$ & 1 & 1 & 0.42 & 0.57 & $1.14 \mathrm{e} 4$ & 241.04 & 239.05 \\
\hline $20,10,5,0$ & 0.50 & 0.55 & 0.32 & 0.60 & 139.36 & 84.66 & 71.00 \\
\hline $40,20,10,20$ & 1 & 1 & 0.47 & 0.51 & $8.69 \mathrm{e} 4$ & $1.08 \mathrm{e} 3$ & $1.08 \mathrm{e} 3$ \\
\hline $40,20,10,10$ & 1 & 1 & 0.42 & 0.47 & $4.61 \mathrm{e} 4$ & $1.04 \mathrm{e} 3$ & $1.04 \mathrm{e} 3$ \\
\hline $40,20,5,10$ & 1 & 1 & 0.42 & 0.58 & $3.03 \mathrm{e} 4$ & 493.10 & 490.95 \\
\hline $40,20,5,0$ & 0.53 & 0.53 & 0.70 & 0.30 & 340.45 & 197.09 & 197.09 \\
\hline $60,30,10,20$ & 1 & 1 & 0.51 & 0.49 & $1.21 \mathrm{e} 5$ & $1.59 \mathrm{e} 3$ & $1.59 \mathrm{e} 3$ \\
\hline $60,30,10,10$ & 1 & 1 & 0.51 & 0.49 & $1.92 \mathrm{e} 5$ & $1.57 \mathrm{e} 3$ & $1.57 \mathrm{e} 3$ \\
\hline $60,30,5,10$ & 1 & 1 & 0.43 & 0.57 & $5.12 \mathrm{e} 4$ & 767.49 & 765.50 \\
\hline $60,30,5,0$ & 0.57 & 0.58 & 0.42 & 0.58 & 552.59 & 276.76 & 222.37 \\
\hline
\end{tabular}

Table 4.1 shows that the minimum values of $f_{1}$ and $f_{3}$ become large as $\beta$ and $\sigma$ become large. Nevertheless, the function values $f_{1}\left(x^{1}\right)$ and $f_{3}\left(x^{3}\right)$ are usually much smaller than $f_{1}(\bar{x})$ and $f_{3}(\bar{x})$, respectively. As to the reliability $\operatorname{rel}_{\epsilon}(x)$ and the expected total loss $\Gamma(x)$, the solutions $x^{1}$ and $x^{3}$ exhibit significantly better performance than $\bar{x}$ as shown in Tables 4.1 and 4.2. Moreover, as to the measure of optimality and feasibility $\pi(\cdot, \cdot)$ which is defined through the stochastic quadratic program (4.1), the solutions $x^{1}$ and $x^{3}$ dominate $\bar{x}$ in most cases. From these results, we may conclude that the ERM formulation yields a solution that has desirable properties in regard to the performance measures related to optimality, feasibility, and reliability.

In most cases in Table 4.2, we find that $\pi\left(x^{1}, x^{3}\right)+\pi\left(x^{3}, x^{1}\right)=1$. This phenomenon may be explained as follows. Let $J=\left\{j \mid \gamma^{j}\left(x^{1}\right)=\gamma^{j}\left(x^{3}\right)\right\}$. By the definition of $\pi(\cdot, \cdot)$, 
we have

$$
\pi\left(x^{1}, x^{3}\right)+\pi\left(x^{3}, x^{1}\right)=1-\sum_{j \in J} p_{j} .
$$

In general, unlike LCP, minimal solutions $x^{1}$ and $x^{3}$ of $f_{1}$ and $f_{3}$ are different, which leads to $\gamma^{j}\left(x^{1}\right) \neq \gamma^{j}\left(x^{3}\right)$ in most of our test problems.

\section{Applications}

In the survey paper by Ferris and Pang [9], many engineering and economic applications of complementarity problems are described. Traffic equilibrium problems constitute one of important applications. Here we use the notations in [9] and the example in [5] to illustrate the model of stochastic LCP and the ERM formulation for traffic equilibrium problems under demand uncertainty.

For a given transportation network with a set of nodes and a set of paths, we suppose the travel demand on origin-destination (OD) pairs is given by a vector $d$ and the users' travel cost function is defined by $A \xi+b$, where $A$ is a positive definite matrix, $b$ is an assigned vector and $\xi$ is the traffic flow. By the Wardrop's user equilibrium principle, the traffic equilibrium problem can be mathematically described as

$$
0 \leq A \xi+b-B^{T} \tau \perp \xi \geq 0, \quad 0 \leq B \xi-d \perp \tau \geq 0,
$$

where $\tau$ represents the minimum transportation costs between OD pairs and $B$ is the path-OD pair incidence matrix.

We consider a simple transportation network [5] shown in Figure 1.

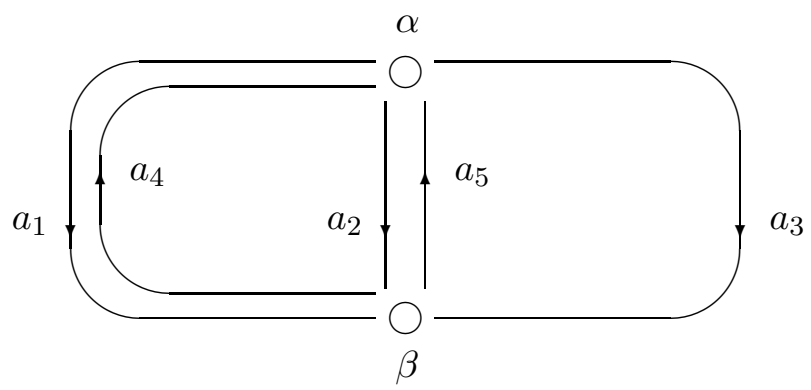

Figure 1: A transportation network

This network consists of two nodes $\{\alpha, \beta\}$ and five paths $\left\{a_{1}, a_{2}, a_{3}, a_{4}, a_{5}\right\}$. Nodes $\alpha$ and $\beta$ are connected by two two-way roads and by one one-way road. Paths $a_{1}, a_{2}, a_{3}$ are directed from $\alpha$ to $\beta$, and $a_{4}, a_{5}$ are directed from $\beta$ to $\alpha$. The travel demand on the direction from $\alpha$ to $\beta$ is 210 , and that on the opposite direction is 120 . The flow on path $a_{i}$ is denoted $\xi_{i}, i=1,2,3,4,5$, and the minimum transportation costs from $\alpha$ to $\beta$ and from $\beta$ to $\alpha$ are denoted $\tau_{1}$ and $\tau_{2}$, respectively. The travel cost function on path $a_{i}$ is 
given by $(A \xi+b)_{i}$, where

$$
A=\left(\begin{array}{ccccc}
10 & 0 & 0 & 5 & 0 \\
0 & 15 & 0 & 0 & 5 \\
0 & 0 & 20 & 0 & 0 \\
2 & 0 & 0 & 20 & 0 \\
0 & 1 & 0 & 0 & 25
\end{array}\right), \quad b=\left(\begin{array}{r}
1000 \\
950 \\
3000 \\
1000 \\
1300
\end{array}\right)
$$

Matrix $B$ and vector $d$ are given by

$$
B=\left(\begin{array}{lllll}
1 & 1 & 1 & 0 & 0 \\
0 & 0 & 0 & 1 & 1
\end{array}\right), \quad d=\left(\begin{array}{l}
210 \\
120
\end{array}\right)
$$

Note that $(B \xi-d)_{1}$ and $(B \xi-d)_{2}$ represent the undelivered transportation demands from $\alpha$ to $\beta$ and from $\beta$ to $\alpha$, respectively. Let

$$
\bar{M}=\left(\begin{array}{cc}
A & -B^{T} \\
B & 0
\end{array}\right), \quad \bar{q}=\left(\begin{array}{c}
b \\
-d
\end{array}\right) .
$$

Then the problem is modeled as the monotone $\operatorname{LCP}(\bar{M}, \bar{q})$, whose unique solution is computed as

$$
\xi=(120,90,0,70,50)^{T}, \text { and } \tau=(2550,2640)^{T} .
$$

The solution shows that for the fixed demand, the one-way road $a_{3}$ has no traffic flow in the equilibrium network. Now the road traffic management office wants to make a road maintenance plan for the coming year. Due to the limit of budget, roads having no certain traffic flow will not be considered. Should road $a_{3}$ be ignored?

Actually, travel demand is not always constant, but may fluctuate regularly with different time periods of day, week and season of the year. Furthermore, the fluctuation further affects the travel cost function, which should be taken into account to get a robust equilibrium model under any realization of the uncertain demand. Therefore, we replace the vector $d$ and matrix $A$ in (5.1) by a perturbed demand vector $d(\omega)$ and a perturbed matrix $A(\omega)$, respectively, and obtain the corresponding random matrix $M(\omega)$ and vector $q(\omega)$ in (5.2). Thus the problem is modeled as $\operatorname{SLCP}(M(\omega), q(\omega))$.

For the network in Figure 1, we assume that the travel demand is given by $d(\omega)=$ $\omega=\left(\omega_{1}, \omega_{2}\right)^{T}$, where $\omega_{1}$ and $\omega_{2}$ follow the truncated normal distribution and the uniform distribution, respectively, as follows:

$$
\omega_{1} \sim 80 \leq \mathcal{N}(210,1200) \leq 340, \quad \omega_{2} \sim \mathcal{U}[60,180]
$$

Define two cost coefficients, which relate to the fluctuation of travel demand, as

$$
c_{1}(\omega)=\frac{\omega_{1}+\omega_{2}}{330}-1, \quad c_{2}(\omega)=\frac{\omega_{1}}{210}-1 .
$$


Let $A(\omega)=A+\tilde{A}(\omega)$ with

$$
\tilde{A}(\omega)=\left(\begin{array}{ccccc}
3 c_{1}(\omega) & 0 & 0 & 0.5 c_{1}(\omega) & 0 \\
0 & 4 c_{1}(\omega) & 0 & 0 & c_{1}(\omega) \\
0 & 0 & 0.5 c_{2}(\omega) & 0 & 0 \\
0 & 0.1 c_{1}(\omega) & 0 & 0 & c_{1}(\omega)
\end{array}\right)
$$

to reflect the situation where the travel cost varies with demand perturbation. Since $a_{3}$ is a one-way road, the influence on $a_{3}$ is much less than on other roads and only relates to the travel demand from $\alpha$ to $\beta$. Moreover, it is easy to find that $E[M(\omega)]=\bar{M}$ and $E[q(\omega)]=\bar{q}$, which means $\operatorname{SLCP}(M(\omega), q(\omega))$ is a monotone SLCP, and a solution of the monotone $\operatorname{LCP}(\bar{M}, \bar{q})$ is also a solution of $\operatorname{EV}$ formulation for $\operatorname{SLCP}(M(\omega), q(\omega))$.

Now, for the ERM formulation (1.4) of $\operatorname{SLCP}(M(\omega), q(\omega))$, we use the sample average approximation method [14] to get the approximation of $f(x)$ as

$$
f^{N}(x):=\frac{1}{N} \sum_{j=1}^{N}\left\|\Phi\left(x, \omega^{j}\right)\right\|^{2},
$$

where $\omega^{j}$ is randomly generated by the given distribution. We use the "min" NCP function to define $\Phi(x, \omega)$ and randomly generate $N=1000$ samples $\left\{\omega^{j}\right\}$. The sample average approximation of the ERM problem $\min _{x \in R_{+}^{7}} f^{N}(x)$ is solved by fmincon in the Matlab (version 7) tool box for constrained optimization. The average of computed solutions for 100 simulations is

$$
\xi=(72,60,39,68,46)^{T}, \text { and } \tau=(2027,2506)^{T} .
$$

Comparing this solution with that of the EV formulation, which is given by (5.3), we find that the transportation flow on path $a_{3}$ is comparable with that on $a_{1}$ and $a_{2}$, and therefore we suggest that $a_{3}$ should not be ignored. One of the reasons may be that although the cost on $a_{3}$ is much higher than those on $a_{1}$ and $a_{2}$ in average, road $a_{3}$ becomes acceptable, or even preferable for users when the travel demand increases, since the increase of cost on road $a_{3}$ is much less than that on $a_{1}$ and $a_{2}$.

\section{Final remark}

The monotone SLCP has a wide range of applications in engineering and economics, and is closely linked to the study of stochastic linear and quadratic programs. Our theoretical and numerical study has revealed that the ERM formulation for the monotone SLCP has various desirable properties. In particular, the ERM formulation produces robust solutions with minimum sensitivity, high reliability, and low risk in violation of feasibility with respect to random parameter variations in SLCP.

Acknowledgement The authors are grateful to three anonymous referees for their many helpful comments. 


\section{References}

[1] J.R. Birge and F. Louveaux, Introduction to Stochastic Programming, Springer-Verlag, New York, 1997.

[2] B. Chen, X. Chen and C. Kanzow, A penalized Fischer-Burmeister NCP-function, Math. Programming, 88 (2000) 211-216.

[3] X. Chen and M. Fukushima, Expected residual minimization method for stochastic linear complementarity problems, Math. Oper. Res. 30 (2005) 1022-1038.

[4] R.W. Cottle, J.-S. Pang, and R.E. Stone, The Linear Complementarity Problem, Academic Press, New York, 1992.

[5] S. Daffermos, Traffic equilibrium and variational inequalities, Transportation Science $\mathbf{1 4}$ (1980), 42-54.

[6] F. Facchinei and J.-S. Pang, Finite-Dimensional Variational Inequalities and Complementarity Problems, I and II, Springer-Verlag, New York, 2003.

[7] H. Fang, X. Chen and M. Fukushima, Stochastic $R_{0}$ matrix linear complementarity problems, Technical Report, 2005-002, Department of Applied Mathematics and Physics, Kyoto

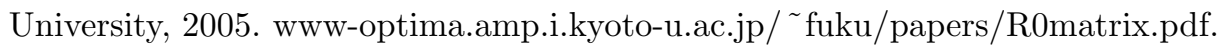

[8] M.C. Ferris, ftp://ftp.cs.wisc.edu/math-prog/matlab/lemke.m, Department of Computer Science, University of Wisconsin, 1998.

[9] M.C. Ferris and J.-S. Pang, Engineering and economic applications of complementarity problems, SIAM Rev., 39 (1997), 669-713.

[10] M. Frank and P. Wolfe, An algorithm for quadratic programming, Nav. Res. Logist., 3 (1956), 95-110.

[11] G. Gürkan, A.Y. Özge and S.M. Robinson, Sample-path solution of stochastic variational inequalities, Math. Programming, 84 (1999), 313-333.

[12] P. Kall and S.W. Wallace, Stochastic Programming, John Wiley \& Sons, Chichester, 1994.

[13] C. Kanzow, N. Yamashita and M. Fukushima, New NCP-functions and their properties, J. Optim. Theory Appl., 94 (1997) 115-135.

[14] A.J. Kleywegt, A. Shapiro, and T. Homen-De-Mello, The sample average approximation method for stochastic discrete optimization, SIAM J. Optim., 12 (2001) 479-502

[15] G.H. Lin and M. Fukushima, New reformulations for stochastic nonlinear complementarity problems, to appear in Optim. Methods Softw.

[16] Z.-Q. Luo and P. Tseng, A new class of merit functions for the nonlinear complementarity problem, in M.C. Ferris and J.-S. Pang (eds.): Complementarity and Variational Problems: State of the Art., SIAM, Philadelphia, PA, 1997, 204-225.

[17] O.L. Mangasarian and J. Ren, New improved error bounds for the linear complementarity problem, Math. Programming, 66 (1994) 241-255.

[18] K. Marti, Stochastic Optimization Methods, Springer-Verlag, Berlin, 2005.

[19] P. Tseng, Growth behavior of a class of merit functions for the nonlinear complementarity problem, J. Optim. Theory Appl., 89 (1996) 17-37.

[20] D. Sun and L. Qi, On NCP-functions, Comp. Optim. Appl., 13 (1999) 201-220. 